\title{
Revisão e Implementação de Soluções Analíticas para a Determinação da Pressão em Poços de Petróleo
}

\section{Igor Caetano Cariello}

Engenharia Mecânica, Instituto Politécnico, UERJ, Brasil.

Paulo de Tarço Honório Jr.

Instituto Politécnico, UERJ, Brasil.

\section{Grazione de Souza}

Departamento de Modelagem Computacional, Instituto Politécnico, UERJ, Brasil.

\section{Helio Pedro Amaral Souto}

Departamento de Modelagem Computacional, Instituto Politécnico, UERJ, Brasil.

Abstract: Well Testing Analysis is a branch of Reservoir Engineering, in which we employ well pressure data from production tests/fluid injection in conjunction with physical-mathematical models to characterize the well-reservoir system, using inverse problems. In these situations, we widely used analytical and semi-analytical solutions of the physical-mathematical model that describes the flow. In this context, the objective of this work is to 1) carry out a bibliographic review on some of the classic analytical solutions for determining the pressure in the producing well and 2) implement the numerical codes for the creation of a computational library, providing the analytical solutions aimed at determining pressure in oil-producing wells. The well-reservoir systems with a vertical well take into account the boundary effects, wellbore storage, formation damage, drawdown and buildup test analysis, and the presence of natural fractures. We obtain the analytical solutions using the Laplace transform and a numerical inversion, using the Stehfest algorithm, to calculate the pressure variation in the time domain.

Key words: Analytical Solutions, Inverve Laplace Transform, Laplace Transform, Stehfest Algorithm, Well Testing Analysis.

Resumo: A Análise de Testes de Poços é um ramo da Engenharia de Reservatórios no qual empregamos dados de pressão de poço a partir de testes de produção/injeção de fluido em conjunto com modelos físico-matemáticos para caracterizar o sistema poço-reservatório, usando problemas inversos. Nessas situações, aplicamos amplamente soluções analíticas e semianalíticas do modelo físico-matemático que descreve o fluxo. Nesse contexto, o objetivo do presente estudo é 1) realizar uma revisão bibliográfica sobre algumas das soluções analíticas clássicas para determinação da pressão no poço produtor e 2) implementar os códigos numéricos para a criação de uma biblioteca computacional, proporcionando as soluções analíticas voltadas para a determinação da pressão em poços produtores de petróleo. Os sistemas poço-reservatório estudados possuem um poço vertical e levam em consideração os efeitos de condições de contorno, a estocagem na coluna de produção do poço, dano à formação, períodos de fluxo e estática, bem como a presença de fraturas naturais. Obtivemos as soluções analíticas usando a transformada de Laplace e uma inversão numérica, utilizando o algoritmo Stehfest, para calcular a variação de pressão ao longo do tempo.

Palavras-chave: Soluções Analíticas, Transformada de Laplace Inversa, Tranformada de Laplace, Algoritmo de Stehfest, Análise de Teste de Poço.

Corresponding author: Grazione de Souza, gsouza@iprj.uerj.br

Received: 20 Oct 2020 / Accepted: 18 Nov 2020 / Published: 20 Dec 2020. 


\section{Introdução}

Na Análise de Testes de Pressão, um dos ramos da Engenharia de Reservatórios de Petróleo [1, 6, 14, 23], temos por meta a caracterização do sistema poço-reservatório através da solução de problemas inversos, utilizando dados de testes de pressão em poços. Desta forma, é possível obtermos as propriedades do sistema poçoreservatório, utilizadas posteriormente como uma estimativa acurada das características do sistema, de forma a permitir uma melhor escolha da estratégia de produção. Em um teste com produção à vazão constante, por exemplo, a pressão no poço cairá com o passar do tempo e estes dados são coletados por um registrador de pressão, alocado em algum ponto do poço. Neste contexto, aplicativos computacionais dedicados à essa área também são utilizados pela indústria do petróleo.

Quando da análise dos testes de pressão utilizamos tipicamente os gráficos especializado e diagnóstico. No gráfico especializado temos a representação da pressão no poço $\left(p_{w f}\right)$ em função do tempo $(t)$, com esse último eixo em escala logarítmica. Os dados obtidos a partir da medição de campo são discretos e contêm ruídos. Por outro lado, o gráfico diagnóstico, com ambos os eixos em escala logarítmica, apresenta uma curva para a queda de pressão no poço e uma outra para a derivada da pressão em relação ao logaritmo neperiano do tempo, $\Delta p_{w f}^{\prime}$, a chamada derivada de Bourdet [4]. Neste trabalho, as linhas contínuas representam a variação de pressão e as tracejadas as derivadas de Bourdet, respectivamente, quando da apresentação de um gráfico diagnóstico. Os perfis das curvas neste gráfico estão relacionados com a sequência de regimes de escoamento que ocorrem e, assim, podem ser empregados na caracterização do sistema poço-reservatório.

De posse de resultados de campo e de um modelo adequado para o escoamento é possível utilizarmos as técnicas de resolução de problemas inversos para determinarmos as propriedades associadas à estocagem no poço [20], à capacidade do reservatório em transportar fluido [17] e ao dano à formação [16], por exemplo.

A partir do conhecimento das propriedades de rocha que formam o reservatório e das características dos fluidos é possível realizar a modelagem do escoamento dos fluidos, mediante a resolução das equações diferenciais parciais que governam o escoamento [19. Nos casos mais próximos da realidade, o escoamento geralmente é descrito por equações diferenciais parciais não-lineares. Portanto, a solução dessas equações, através dos métodos analíticos, está restrita a casos particulares. Entretanto, isso não inviabiliza o seu uso, visto que as soluções analíticas, mesmo obtidas com hipóteses simplificadoras, auxiliam:

1. no entendimento da física do escoamento;

2. no estudo de problemas simplificados;

3. na caracterização do sistema poços-reservatório (problemas inversos);
4. na verificação de simuladores numéricos (testados em problemas particulares);

5. na utilização conjunta, em alguns casos, com os simuladores numéricos.

$\mathrm{Na}$ literatura, podemos encontrar soluções analíticas clássicas, consolidadas, para a determinação da pressão no poço em escoamentos em reservatórios, como as revisadas por Chaudhry [6, Dake 9, Ozkan [22] e Rosa et al [26]. No entanto, ao longo do tempo, esforços têm sido realizados para reduzirmos o número de hipóteses simplificadoras e foram desenvolvidas soluções analíticas/semianalíticas que podem ser aplicadas aos casos mais realísticos, tais como as estratégias apresentadas por Chen et al. 11, Du et al. [12, Ozkan 22], Qiu e Li, [25], Wendong et al. [29] e Zeng e Zhao [30].

Diferentes técnicas têm sido empregadas na obtenção das soluções das equações diferenciais que governam o escoamento no reservatório como, por exemplo, a Transformada de Laplace e a Transformada Inversa de Laplace [27. Em geral, a menos que hipóteses simplificadoras sejam adotadas, devemos utilizar uma técnica numérica para obtermos a transformada inversa.

Devemos destacar que Hassanzadeh e PooladiDarvish 15] realizaram um estudo comparativo de algumas das técnicas de inversão numérica e comprovaram a eficiência do Algoritmo de Stehfest (utilizado neste trabalho). Exemplos de aplicações envolvendo a Transformada de Laplace e a sua inversão numérica podem ser encontradas em [2, 3, 5, 8.

Este trabalho tem como objetivos principais a revisão e a implementação de códigos numéricos voltados para a obtenção de um conjunto de soluções analíticas para a determinação da pressão em poços verticais produtores de óleo, visando à criação de uma biblioteca de códigos para a sua consequente utilização no Laboratório de Modelagem Multiescala e Transporte de Partículas (www.labtran.iprj.uerj.br) da Universidade do Estado do Rio de Janeiro.

\section{Escoamento monofásico em meio poroso}

Para o escoamento monofásico em um meio poroso o balanço de massa é dado pela equação da continuidade [13]

$$
\frac{\partial}{\partial t}(\phi \rho)+\nabla \cdot(\rho \mathbf{v})-q_{m}=0,
$$

onde $\phi$ é a porosidade efetiva, $\rho$ é a massa específica, $\mathbf{v}$ é a velocidade aparente do fluido e $q_{m}$ um termo de fonte/sorvedouro.

Por outro lado, a equação que expressa a conservação da quantidade de movimento é a lei clássica de Darcy [13],

$$
\mathbf{v}=-\frac{\mathbf{k}}{\mu}(\nabla p-\gamma \nabla Z),
$$


onde $\mathbf{k}$ é o tensor de permeabilidade absoluta do meio poroso, $\mu$ é a viscosidade do fluido, $p$ é a pressão, $\gamma=\rho g$ ( $g$ é a magnitude da aceleração da gravidade) e $\nabla Z$ é o gradiente de profundidade. A lei de Darcy clássica é estritamente válida para um conjunto específico de hipóteses 13].

A partir das equações da continuidade e da lei de Darcy clássica, podemos obter uma equação para a determinação da distribuição de pressão no reservatório,

$$
\nabla \cdot\left(\frac{\mathbf{k} \nabla p}{\mu B}\right)=\left(\frac{\phi c}{B^{0}}+\frac{\phi^{0} c_{\phi}}{B}\right) \frac{\partial p}{\partial t}
$$

tendo sido desprezados o efeito da gravidade e o termo fonte e assumimos que $\phi=\phi(p)$ e $B=B(p)$,

$$
\begin{aligned}
& B=\frac{B^{0}}{1+c\left(p-p^{0}\right)}, \\
& \phi=\frac{\phi^{0}}{1+c_{\phi}\left(p-p^{0}\right)},
\end{aligned}
$$

onde nessas equações $B$ é o Fator Volume Formação $(\mathrm{FVF})$, que é a razão entre o volume do fluido na pressão e temperatura do reservatório e o volume ocupado pelo mesmo em condições padrão de temperatura e pressão, $c$ é o coeficiente de compressibilidade isotérmica do fluido, $c_{\phi}$ é o coeficiente de compressibilidade da rocha e o sobrescrito 0 indica os valores de referência. Para maiores detalhes sobre propriedades de rochas e fluidos recomenda-se ao leitor o livro de Dandekar [10.

Consideramos, aqui, as soluções para o escoamento unidimensional na direção radial [26, 22, levando em conta que a permeabilidade e a viscosidade são constantes. Inicialmente, mostraremos as equações para o sistema de coordenadas Cartesianas e, posteriormente, usaremos o sistema de coordenadas cilíndricas. Então,

$$
\frac{k}{\mu} \frac{\partial}{\partial x}\left(\frac{1}{B} \frac{\partial p}{\partial x}\right)=\left(\frac{\phi c}{B^{0}}+\frac{\phi^{0} c_{\phi}}{B}\right) \frac{\partial p}{\partial t}
$$

A partir do lado esquerdo da Eq. 6,

$$
\frac{\partial}{\partial x}\left(\frac{1}{B} \frac{\partial p}{\partial x}\right)=\frac{1}{B} \frac{\partial^{2} p}{\partial x^{2}}+\frac{c}{B^{0}}\left(\frac{\partial p}{\partial x}\right)^{2} .
$$

Considerando-se baixos gradientes de pressão e o baixo valor de $c$,

$$
c\left(\frac{\partial p}{\partial x}\right)^{2}<<\frac{1}{B} \frac{\partial^{2} p}{\partial x^{2}}
$$

e, substituindo a Eq. (7) na Eq. (6), a luz da Eq. (8), tem-se,

$$
\frac{\partial^{2} p}{\partial x^{2}}=\frac{\mu B}{k}\left(\frac{\phi c}{B^{0}}+\frac{\phi^{0} c_{\phi}}{B}\right) \frac{\partial p}{\partial t} .
$$

Reorganizando o lado direito da Eq. (9), utilizando-se as equações para FVF e porosidade,

$$
\frac{\mu B}{k}\left(\frac{\phi c}{B^{0}}+\frac{\phi^{0} c_{\phi}}{B}\right) \frac{\partial p}{\partial t}=
$$

$$
\frac{\mu \phi}{k}\left(\frac{c}{\left[1+c\left(p-p^{o}\right)\right]}+\frac{c_{\phi}}{\left[1+c_{\phi}\left(p-p^{o}\right)\right]}\right) \frac{\partial p}{\partial t} .
$$

Considerando $c\left(p-p^{o}\right)$ e $c_{\phi}\left(p-p^{o}\right)$ como valores baixos e definindo $c_{T}=\left(c+c_{\phi}\right)$, a compressibilidade total, substitui-se a Eq. (10) na Eq. (9). Assim,

$$
\frac{\partial^{2} p}{\partial x^{2}}=\frac{\mu \phi c_{T}}{k} \frac{\partial p}{\partial t} .
$$

onde $c_{T}=\left(c+c_{\phi}\right)$ representa o coeficiente de compressibilidade total e a sua correspondente forma em coordenadas cilíndricas é dada por:

$$
\frac{1}{r} \frac{\partial}{\partial r}\left(r \frac{\partial p}{\partial r}\right)=\frac{1}{\eta} \frac{\partial p}{\partial t},
$$

onde $\eta=k /\left(\phi \mu c_{T}\right)$ é a difusividade hidráulica.

Na resolução da Eq. 12 impomos uma condição inicial dada por $p\left(r, t_{0}\right)=p_{i}$, onde $p_{i}$ é a pressão inicial no reservatório.

No que diz respeito às condições de contorno, uma condição de contorno interna para a pressão é prescrita no raio do poço, $r=r_{w}$.

Nas fronteiras externas, uma condição de contorno do tipo Dirichlet conduz a uma pressão prescrita na fronteira. Tal condição de contorno é utilizada em reservatórios constantemente alimentados por um forte influxo devido à existência de um aquífero, de forma que a pressão na fronteira entre o reservatório e o aquífero permaneça constante.

Em contrapartida, quando prescrevemos o fluxo mássico através da fronteira do reservatório, temos uma condição de contorno do tipo Neumann. A especificação de um fluxo normal à fronteira corresponde à prescrição de um gradiente de pressão na direção normal à fronteira. Em fronteiras seladas (impermeáveis) não há fluxo, de modo que impomos um gradiente de pressão nulo [13.

\section{Soluções revisadas para a pressão no poço}

Nesta seção, revisamos cinco soluções analíticas conhecidas para a determinação da pressão em poços produtores. Três são obtidas a partir da imposição de diferentes condições de contorno, uma é utilizada quando há mudança na produção ao longo do tempo e a última leva em conta as heterogeneidades do reservatório.

\subsection{Caso 1}

Primeiro, vamos estudar o caso no qual o reservatório é considerado infinito, homogêneo e com espessura uniforme; o regime é transiente; o escoamento é unidirecional na direção $r$; e o poço vertical penetra totalmente a formação rochosa.

A variação de pressão é definida como

$$
\Delta p(r, t)=p_{i}-p(r, t),
$$


de forma que é possível reescrever a Eq. (12) como

$$
\frac{1}{r} \frac{\partial}{\partial r}\left(r \frac{\partial \Delta p}{\partial r}\right)=\frac{1}{\eta} \frac{\partial \Delta p}{\partial t} .
$$

e, então, a condição inicial é dada por

$$
\Delta p(r, t=0)=0 .
$$

enquanto que para a condição de contorno externa temos que

$$
\Delta p(r \rightarrow \infty, t)=0 .
$$

Para uma taxa de produção constante, $Q_{s c}$, em condições padrão do poço, impomos a seguinte condição de contorno interna

$$
\left(r \frac{\partial \Delta p}{\partial r}\right)_{r=r_{w}}=-\frac{Q_{s c} B \mu}{2 \pi k h} .
$$

Em seguida, tomamos a Transformada de Laplace 22] de modo a obtermos para condição de contorno externa

$$
\overline{\Delta p}(r \rightarrow \infty, s)=0,
$$

para a condição de contorno interna

$$
\left(r \frac{d \overline{\Delta p}}{d r}\right)_{r=r_{w}}=-\frac{Q_{s c} B \mu}{2 \pi k h s}
$$

e, para a equação diferencial parcial governante [22],

$$
\frac{1}{r} \frac{d \overline{\Delta p}}{d r}+\frac{d^{2} \overline{\Delta p}}{d r^{2}}-\frac{s}{\eta} \overline{\Delta p}=0 .
$$

Como a Eq. 20 pode ser escrita na forma da equação modificada de Bessel, a sua solução geral é dada por [22

$$
\overline{\Delta p}(s)=C_{1} I_{0}(r \sqrt{s / \eta})+C_{2} K_{0}(r \sqrt{s / \eta}),
$$

onde $I_{0}$ e $K_{0}$ são as funções modificadas de Bessel [22].

Da condição de contorno externa e sabendo que $\lim _{r \rightarrow 0} I_{0}(r)=\infty$ 22, chegamos a conclusão de que $C_{1}=0$ para que a condição de contorno seja satisfeita. Então,

$$
\overline{\Delta p}(s)=C_{2} K_{0}(r \sqrt{s / \eta})
$$

Para encontrarmos o valor de $C_{2}$ utilizamos a condição de contorno interna,

$$
\begin{aligned}
\left(r \frac{d \overline{\Delta p}}{d r}\right)_{r=r_{w}} & =-C_{2} r_{w} \sqrt{s / \eta} K_{1}\left(r_{w} \sqrt{s / \eta}\right) \\
& =-\frac{Q_{s c} B \mu}{2 \pi k h s}
\end{aligned}
$$

ou seja,

$$
C_{2}=\frac{Q_{s c} B \mu}{2 \pi k h} \frac{1}{s r_{w} \sqrt{s / \eta} K_{1}\left(r_{w} \sqrt{s / \eta}\right)}
$$

e a forma final da solução é dada por

$$
\overline{\Delta p}(s)=\frac{Q_{s c} B \mu}{2 \pi k h} \frac{K_{0}(r \sqrt{s / \eta})}{s r_{w} \sqrt{s / \eta} K_{1}\left(r_{w} \sqrt{s / \eta}\right)} .
$$

A solução no domínio do tempo pode ser obtida de duas formas: através de uma aproximação ainda no domínio de Laplace, que leve a uma forma passível de inversão via uma Tabela de Transformada Inversa de Laplace (solução da linha fonte) [22], ou por uma inversão numérica [15, 27, como neste trabalho.

\subsection{Caso 2}

Tratamos, agora, do caso do escoamento em um reservatório cilíndrico e selado, com uma pressão inicial uniforme, em regime transiente, com um poço vertical penetrando totalmente na formação e com uma taxa de produção constante. Como o reservatório é selado,

$$
\left(\frac{d \overline{\Delta p}}{d r}\right)_{r=r_{e}}=0
$$

e, portanto, da Eq. 21] obtemos

$$
\begin{aligned}
\left(\frac{d \overline{\Delta p}}{d r}\right)_{r=r_{e}} & =C_{1} \sqrt{s / \eta} I_{1}\left(r_{e} \sqrt{s / \eta}\right) \\
& -C_{2} \sqrt{s / \eta} K_{1}\left(r_{e} \sqrt{s / \eta}\right)=0,
\end{aligned}
$$

onde $I_{1}$ também é uma função de Bessel modificada. Reescrevendo o resultado em função de $C_{2}$,

$$
C_{2}=C_{1} \frac{I_{1}\left(r_{e} \sqrt{s / \eta}\right)}{K_{1}\left(r_{e} \sqrt{s / \eta}\right)}
$$

e substituindo-o na Eq. 21,

$$
\begin{aligned}
\overline{\Delta p}(s) & =C_{1} I_{0}(r \sqrt{s / \eta}) \\
& +C_{1} \frac{K_{0}(r \sqrt{s / \eta}) I_{1}\left(r_{e} \sqrt{s / \eta}\right)}{K_{1}\left(r_{e} \sqrt{s / \eta}\right)}
\end{aligned}
$$

Agora, da condição de contorno interna, Eq. (17),

$$
C_{1}=\frac{A_{1} K_{1}\left(r_{e} \sqrt{s / \eta}\right)}{A_{2}-A_{3}}
$$

onde

$$
\begin{aligned}
& A_{1}=\frac{Q_{s c} B \mu}{2 \pi k h} \frac{1}{s \sqrt{s / \eta} r_{w}} \\
& A_{2}=I_{1}\left(\sqrt{s / \eta} r_{e}\right) K_{1}\left(\sqrt{s / \eta} r_{w}\right) \\
& A_{3}=I_{1}\left(\sqrt{s / \eta} r_{w}\right) K_{1}\left(\sqrt{s / \eta} r_{e}\right)
\end{aligned}
$$

CALIBRE Vol.5, Suplemento - Dezembro, 2020 
Então, após substituição da expressão de $C_{1}$ na Eq. (28), $\quad$ onde

$$
C_{2}=\frac{A_{1} I_{1}\left(r_{e} \sqrt{s / \eta}\right)}{A_{2}-A_{3}}
$$

Finalmente, das Eqs. (30), 344 e 21) obtemos a solução procurada

$$
\overline{\Delta p}(s)=A_{1}\left(\frac{A_{4}+A_{5}}{A_{2}-A_{3}}\right),
$$

onde

$$
\begin{aligned}
& A_{4}=I_{0}(r \sqrt{s / \eta}) K_{1}\left(r_{e} \sqrt{s / \eta}\right), \\
& A_{5}=I_{1}\left(r_{e} \sqrt{s / \eta}\right) K_{0}(r \sqrt{s / \eta}) .
\end{aligned}
$$

\subsection{Caso 3}

À exceção da estocagem no poço e do dano à formação, as mesmas características do Caso 1 são compartilhadas com o Caso 3.

$\mathrm{Na}$ estocagem, o fluido presente inicialmente na coluna de produção é aquele que é primeiramente produzido. O coeficiente de estocagem, $C$, é dado por $\pi r_{w}^{2} L_{w f} c$, onde $L_{w f}$ é o comprimento do poço contento o fluido no instante inicial.

Na prática, também, costuma ocorrer o dano à formação, que leva ao surgimento de uma camada, ao redor do poço, com um valor de permeabilidade reduzido. Como consequência, existe uma dificuldade adicional à passagem de fluido. Segundo Rosa et al [26], o seu efeito é percebido através de uma variação de pressão acentuada perto do poço. O fator de skin, $S$, é um número adimensional usado na modelagem do dano à formação e ele é dado por [26]

$$
S=\left(\frac{k}{k_{s}}-1\right) \ln \left(\frac{r_{w}}{r_{s}}\right)
$$

onde $k_{s}$ é a permeabilidade na região delimitada pelo raio $r_{s}$.

Definindo o dano a partir da condição de contorno interna, para uma vazão na face da formação igual a $Q_{s f}$,

$$
S=\frac{p\left(r_{w}^{+}\right)-p_{w f}}{\left(r \frac{\partial p}{\partial r}\right)_{r=r_{w}}}=\frac{p\left(r_{w}^{+}\right)-p_{w f}}{\frac{Q_{s f} B \mu}{2 \pi k h}},
$$

onde $p\left(r_{w}^{+}\right)$é a pressão imediatamente após a zona de dano e $p_{w f}$ é a pressão no interior do poço. Portanto,

$$
\Delta p_{w f}=\Delta p\left(r_{w}^{+}\right)-S\left(r \frac{\partial \Delta p}{\partial r}\right)_{r=r_{w}} .
$$

Quando utilizamos a vazão de superfície, devemos considerar que o reservatório pode estar acumulando ou perdendo fluido e, por isso,

$$
Q_{s c}=Q_{w b}+Q_{s f}
$$

$$
Q_{w b}=\frac{C}{B} \frac{d}{d t} \Delta p_{w f},
$$

enquanto que $Q_{s f}$ é a taxa de produção na superfície de contato com a rocha,

$$
Q_{s f}=-\frac{2 \pi k h}{B \mu}\left(r \frac{\partial \Delta p}{\partial r}\right)_{r=r_{w}} .
$$

Assim, das Eqs. 41], 42 e 443,

$$
\frac{C}{B} \frac{d}{d t} \Delta p_{w f}-\frac{2 \pi k h}{B \mu}\left(r \frac{\partial \Delta p}{\partial r}\right)_{r=r_{w}}=Q_{s c} .
$$

Aplicando, em seguida, a transformada de Laplace às Eqs. 40 e 44, respectivamente,

$$
\overline{\Delta p}_{w f}=\overline{\Delta p}\left(r_{w}^{+}\right)-S\left(r \frac{d \overline{\Delta p}}{d r}\right)_{r=r_{w}}
$$

$\mathrm{e}$

$$
\frac{C}{B} s \overline{\Delta p}_{w f}-\frac{2 \pi k h}{B \mu}\left(r \frac{d \overline{\Delta p}}{d r}\right)_{r=r_{w}}=\frac{Q_{s c}}{s} .
$$

Analogamente ao que foi feito no Caso 1, sabemos que a solução geral do escoamento assume a forma da Eq. 222. Por conseguinte, a sua utilização permite que escrevamos a Eq. 45) na forma

$$
\begin{aligned}
\overline{\Delta p}_{w f} & =C_{2} K_{0}\left(r_{w}^{+} \sqrt{s / \eta}\right) \\
& +C_{2} S r_{w} \sqrt{s / \eta} K_{1}\left(r_{w} \sqrt{s / \eta}\right) .
\end{aligned}
$$

e, da sua substituição na Eq. 46,

$$
\begin{aligned}
& C_{2} \frac{C}{B} s\left[K_{0}\left(r_{w}^{+} \sqrt{s / \eta}\right)+S r_{w} \sqrt{s / \eta} K_{1}\left(r_{w} \sqrt{s / \eta}\right)\right] \\
+ & C_{2}\left(\frac{2 \pi k h}{B \mu}\right) r_{w} \sqrt{s / \eta} K_{1}\left(r_{w} \sqrt{s / \eta}\right)=\frac{Q_{s c}}{s} .
\end{aligned}
$$

Então, a partir dessa equação é possível encontrarmos o valor de $C_{2}$,

$$
C_{2}=\frac{A_{6}}{A_{7}+A_{8}}
$$

onde

$$
\begin{aligned}
A_{6} & =\frac{Q_{s c} B \mu}{2 \pi k h s} \\
A_{7} & =r_{w} \sqrt{s / \eta} K_{1}\left(r_{w} \sqrt{s / \eta}\right) \\
A_{8} & =\frac{C \mu s}{2 \pi k h}\left[K_{0}\left(r_{w}^{+} \sqrt{s / \eta}\right)\right. \\
& \left.+S r_{w} \sqrt{s / \eta} K_{1}\left(r_{w} \sqrt{s / \eta}\right)\right]
\end{aligned}
$$

Por fim, substituindo a Eq. 49, na Eq. 47,,

$$
\begin{aligned}
\overline{\Delta p}_{w f} & =\frac{A_{6} K_{0}\left(r_{w}^{+} \sqrt{s / \eta}\right)}{A_{7}+A_{8}} \\
& +\frac{A_{6} S r_{w} \sqrt{s / \eta} K_{1}\left(r_{w} \sqrt{s / \eta}\right)}{A_{7}+A_{8}} .
\end{aligned}
$$




\subsection{Caso 4}

Neste caso, o reservatório e o escoamento possuem as mesmas características que as do Caso 3. Contudo, agora, um outro teste de pressão é idealizado, no qual o poço fica fechado por um período de tempo, com o intuito de estabelecermos um estágio de equalização de pressão. Assim sendo, um novo tempo é introduzido no problema, $t_{p}$, que corresponde ao tempo no qual o poço ficou aberto. Logo, para o Caso 4, admitimos um teste pressão envolvendo períodos de produção e fechamento do poço [22].

Modificando a Eq. (44) para que ela possa ser aplicada ao problema em questão

$$
\begin{aligned}
& \frac{C}{B} \frac{d}{d t} \Delta p_{w f}-\frac{2 \pi k h}{B \mu}\left(r \frac{\partial \Delta p}{\partial r}\right)_{r=r_{w}} \\
& =Q_{s c}\left[1-H\left(t-t_{p}\right)\right]
\end{aligned}
$$

onde $H$ representa a função unitária de Heaviside. Tomando a sua transformada de Laplace obtemos [22],

$$
\begin{aligned}
& \frac{C}{B} s \overline{\Delta p}_{w f}-\frac{2 \pi k h}{B \mu}\left(r \frac{\partial \overline{\Delta p}}{\partial r}\right) \\
& =\frac{Q_{s c}}{s}\left(1-e^{-s t_{p}}\right) .
\end{aligned}
$$

Dando prosseguimento, substituímos a Eq. 47 na Eq. 55,

$$
\begin{gathered}
C_{2} \frac{C}{B} s\left[K_{0}\left(r_{w}^{+} \sqrt{s / \eta}\right)+S r_{w} \sqrt{s / \eta} K_{1}\left(r_{w} \sqrt{s / \eta}\right)\right] \\
+C_{2}\left(\frac{2 \pi k h}{B \mu}\right) r_{w} \sqrt{s / \eta} K_{1}\left(r_{w} \sqrt{s / \eta}\right)=\frac{Q_{s c}}{s}\left(1-e^{s t_{p}}\right) .
\end{gathered}
$$

Em seguida, explicitamos $C_{2}$ :

$$
C_{2}=\frac{A_{6}\left(1-e^{-s t_{p}}\right)}{A_{7}+A_{9}}
$$

onde

$$
A_{9}=\frac{C \mu s}{2 \pi k h}\left[K_{0}\left(r_{w}^{+} \sqrt{s / \eta}\right)+S A_{7}\right]
$$

Por último, o valor de $C_{2}$ é introduzido na Eq. (47),

$$
\overline{\Delta p}_{w f}=\frac{A_{6}\left[K_{0}\left(\sqrt{s / \eta} r_{w}+\right)+S A_{7}\right]\left(1-e^{-s t_{p}}\right)}{A_{7}+A_{9}}
$$

Devido a uma descontinuidade, causada por $\left(1-e^{-s t_{p}}\right)$ quando $t=t_{p}$, na inversão numérica é recomendada a utilização da técnica sugerida por Chen e Raghavan [7,

$$
\begin{aligned}
L^{-1}\left\{\bar{f}(s)\left(1-e^{-s t_{p}}\right)\right\}_{t} & =L^{-1}\{\bar{f}(s)\}_{t} \\
& -L^{-1}\{\bar{f}(s)\}_{t-t_{p}},
\end{aligned}
$$

realizando, assim, a inversão numérica termo a termo.

\subsection{Caso 5}

No Caso 5, recuperamos a solução proposta por Warren e Root [28] para os meios porosos naturalmente fraturados.

As fraturas naturais surgem nas rochas devido aos diferentes esforços exercidos sobre elas (causando deformações físicas nas suas estruturas) ou devido à ação de agentes químicos. As fraturas modificam as propriedades do reservatório, fazendo com que a porosidade e a permeabilidade sejam diferentes nas regiões fraturada e não fraturada. Portanto, elas modificam o escoamento dos fluidos na matriz porosa, resultando em um aumento da produção do reservatório uma vez que, em geral, a região fraturada possui valores mais elevados da permeabilidade e da porosidade [26].

Ao se iniciar a produção em um reservatório naturalmente fraturado, o escoamento será caracterizado por quatro regimes. Inicialmente, a produção é proveniente do escoamento através apenas das fraturas. Depois, ocorre um período de transição até que, na sequência, tanto a região fraturada quanto a não fraturada irão contribuir para a produção no reservatório. Por último, podem aparecer os efeitos de fronteira [22].

O Caso 5 contempla a solução do escoamento em um reservatório infinito, naturalmente fraturado, com uma pressão inicial uniforme e uma produção a vazão constante. A solução apresentada está baseada no trabalho desenvolvido por Warren e Root 28] e é aplicada tendo em vista o modelo de camadas de Kazemi [18] (Figura 1.

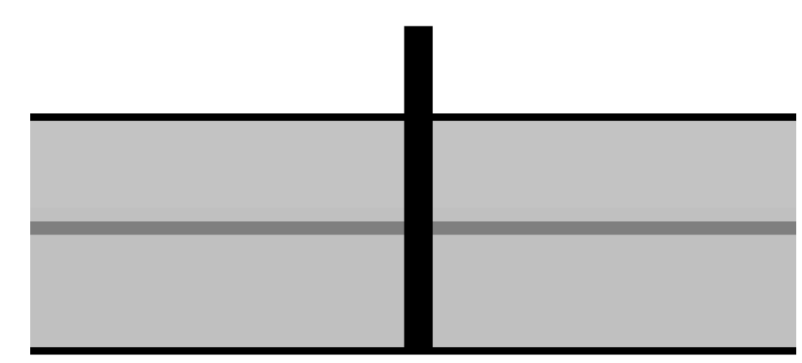

Figura 1: Em preto o poço, em cinza escuro a fratura e em cinza claro a matriz.

O detalhamento para a obtenção da solução é omitido, sabendo que o mesmo pode ser visto em [28]. Portanto, no domínio de Laplace, a variação de pressão é calculada a partir da equação

$$
\overline{\Delta p}(s)=\frac{K_{0}(\sqrt{s f(s)})}{s \sqrt{s f(s)} K_{1}(\sqrt{s f(s)})}
$$

onde $f(s)$ é definida como

$$
f(s)=\frac{\omega(1-\omega) s+\lambda}{(1-\omega) s+\lambda},
$$

sendo $\lambda$ o parâmetro que reflete a relacão do fluxo de fluido entre a matriz porosa e a fratura e $\omega$ o parâmetro

CALIBRE Vol.5, Suplemento - Dezembro, 2020. 
que mede a relação entre a quantidade de fluido armazenado na fratura e no sistema fratura mais matriz porosa (maiores detalhes sobre a determinação dos valores adimensionais $\lambda$ e $\omega$ podem ser obtidos em [1]).

\section{Implementação}

Na Figura (2), vemos como variam as funções modificadas de Bessel $I_{0}, I_{1}, K_{0}$ e $K_{1}$. Conforme pudemos ver das soluções aqui revisadas, essas funções combinadas nos permitem calcularmos os perfis de queda de pressão nos poços produtores. Para os maiores valores de $s$ obtemos a variação de pressão para os tempos curtos, enquanto que para os menores valores reproduzimos o seu comportamento decorrido um maior intervalo de tempo no domínio real.

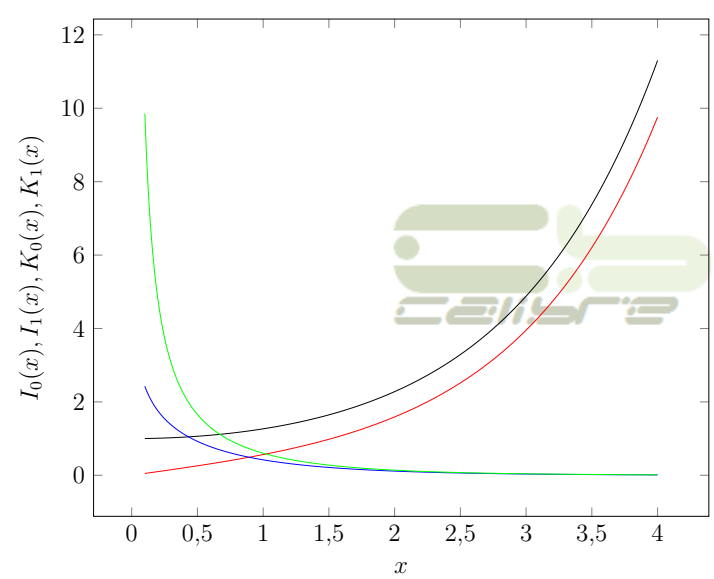

Figura 2: Funções modificadas de Bessel para $0,1 \leq x \leq 4$ : $I_{0}(x)$ em preto, $I_{1}(x)$ em vermelho, $K_{0}(x)$ em azul e $K_{1}(x)$ em verde.

Como o estudo dos efeitos da estocagem, do dano à formação e do escoamento transiente são muito importantes na Análise de Testes de Pressão, é essencial que possamos avaliar as funções modificadas de Bessel para os maiores valores de $s$.

Neste trabalho, o cálculo das funções modificadas de Bessel é feito a partir das rotinas sugeridas em [24]. Nelas, é possível encontrar as relações de recorrência existente entre as funções, com $K_{0}$ sendo calculada empregando $I_{0}$ e $K_{1}$ uma vez conhecida $I_{1}$.

Nos códigos numéricos implementados as funções e as variáveis foram declaradas como tendo dupla precisão, de modo que os ruídos oriundos do uso da precisão simples foram suprimidos e a faixa máxima para o valor do argumento das funções modificadas de Bessel passou de 95 para cerca de 600. Portanto, soluções mais acuradas puderam ser obtidas para tempos mais curtos no domínio do tempo. Além disso, não ocorreram problemas no que diz respeito à representação das derivadas de Bourdet.

As soluções são obtidas, conforme já visto, no domínio de Laplace. Portanto, a menos que algumas sim- plificações sejam feitas, não encontramos as transformadas inversas de Laplace para as soluções aqui estudadas. Como consequência, somos obrigados a realizar a transformação inversa numericamente, a fim de obtermos a solução no domínio do tempo. Optamos, neste trabalho, por construir a biblioteca contendo as soluções analíticas mediante a utilização do algoritmo de Stehfest [27. Esse algoritmo é suficientemente acurado, de modo que o mesmo é empregado na resolução de muitos problemas na engenharia de petróleo, onde a sua aplicação é muito difundida.

No algoritmo de Stehfest a função inversa é calculada por, já utilizando as variáveis em termos da variação de pressão,

$$
\Delta p(t)=\frac{\ln (2)}{t} \sum_{i=1}^{N} V_{i} \overline{\Delta p}\left(\frac{\ln (2)}{t} i\right)
$$

onde $N$ é a quantidade de termos. Para problemas de escoamento utilizamos os valores pares no intervalo $6 \leq$ $N \leq 18$. Os valores de $V_{i}$ variam de acordo com a quantidade de termos e são determinados através da fórmula

$$
V_{i}=(-1)^{\left(\frac{N}{2}\right)+i} \alpha,
$$

onde

$$
\alpha=\sum_{k=\frac{i+1}{2}}^{\min \left(i, \frac{N}{2}\right)} \frac{k^{\frac{N}{2}}(2 k) !}{\left(\frac{N}{2}-k\right) ! k !(k-1) !(i-k) !(2 k-i) !}
$$

Como exemplo, a Tabela 1 mostra os valores de $V_{i}$ para $N=10$. No código implementado é possível trabalharmos com os seguintes valores: $N=8,10,12,14 \mathrm{e}$ 16.

Tabela 1 Coeficientes de $V_{i}$ para $N=10$

\begin{tabular}{ll}
\hline$V_{i}$ & Valor \\
\hline$V_{1}$ & 0,08333 \\
$V_{2}$ & $-32,08333$ \\
$V_{3}$ & $1.279,0$ \\
$V_{4}$ & $-15.623,666$ \\
$V_{5}$ & $84.244,1666$ \\
$V_{6}$ & $-23.6957,5$ \\
$V_{7}$ & $375.911,666$ \\
$V_{8}$ & $-340.071,666$ \\
$V_{9}$ & $164.062,5$ \\
$V_{10}$ & $-32.812,5$ \\
\hline
\end{tabular}

A derivada de Bourdet 4, utilizada no gráfico diagnóstico, auxilia na identificação dos diferentes regimes de escoamento presentes no sistema poço-reservatório, ao evidenciar as mudanças na variação do comportamento da queda de pressão no poço. Existem diferentes fórmulas para a determinação dessa derivada. Aqui, adotamos o esquema a três pontos [21]

$$
\left(\frac{d \Delta p_{w f}}{d \ln \Delta t}\right)_{j}=\Delta p_{w f}^{\prime}=B_{1}+B_{2}, \quad j \geq 2,
$$


onde

$$
B_{1}=\frac{\Delta p_{j}-\Delta p_{L}}{\ln \left(\Delta t_{j} / \Delta t_{L}\right)} \frac{\ln \left(\Delta t_{R} / \Delta t_{j}\right)}{\ln \left(\Delta t_{R} / \Delta t_{L}\right)}
$$

e

$$
B_{2}=\frac{\Delta p_{R}-\Delta p_{j}}{\ln \left(\Delta t_{R} / \Delta t_{j}\right)} \frac{\ln \left(\Delta t_{j} / \Delta t_{L}\right)}{\ln \left(\Delta t_{R} / \Delta t_{L}\right)}
$$

onde $L=j-1$, e $R=j+1$, de forma que não calculamos a derivada no primeiro e no último tempo do registro de pressão.

Contudo, chamamos a atenção do leitor para o fato de que existem versões que utilizam dois pontos e outras que empregam a filtragem de dados, para que nem todos as informações sejam utilizadas no cálculo da derivada, com o objetivo de eliminar os ruídos quando da determinação da derivada para dados de campo [21].

\section{Resultados}

A Tabela 2 apresenta os dados que foram adotados como parâmetros padrão para a determinação da variação da queda de pressão para o Caso 1. Entretanto, algumas alterações podem ser feitas quando da análise de sensibilidade.

\begin{tabular}{|c|c|}
\hline Dado & Valor \\
\hline$B$ & $1,50 \mathrm{~m}^{3} / \mathrm{std} \mathrm{m}^{3}$ \\
\hline$c$ & $1,00^{-8} \mathrm{~Pa}^{-1}$ \\
\hline$c_{\phi}$ & $1,00^{-8} \mathrm{~Pa}^{-1}$ \\
\hline$h$ & $20,00 \mathrm{~m}$ \\
\hline$k$ & $10,00^{-13} \mathrm{~m}^{2}$ \\
\hline$N$ & 10 \\
\hline$p_{i}$ & $30,00 \times 10^{6} \mathrm{~Pa}$ \\
\hline$Q_{s c}$ & 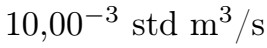 \\
\hline$r$ & $0,10 \mathrm{~m}$ \\
\hline$r_{w}$ & $0,10 \mathrm{~m}$ \\
\hline$\mu$ & $0,01 \mathrm{~Pa} \cdot \mathrm{s}$ \\
\hline$\phi$ & 0,20 \\
\hline
\end{tabular}

Tabela 2 Parâmetros do Caso 1

Na Figura 3 apresentamos o gráfico diagnóstico para o problema do escoamento para o Caso 1. Nele, é possível ver que capturamos o regime transiente. Observamos, também, que a derivada é representada na forma de um patamar, típico da encontrada para este tipo de escoamento em um gráfico diagnóstico [1.

Em seguida, obtivemos os resultados para o Caso 1 variando os valores da permeabilidade. Os resultados são mostrados nas Figuras 4 e 5 . Conforme o esperado, quanto maior for a permeabilidade, menor será a queda de pressão necessária para que ocorra o escoamento para uma vazão de produção fixa. Por outro lado, quando a permeabilidade diminui, a queda de pressão deve aumentar de modo a suplementar a energia necessária para que o fluido se mova no reservatório apresentando, agora,

CALIBRE Vol.5, Suplemento - Dezembro, 2020.

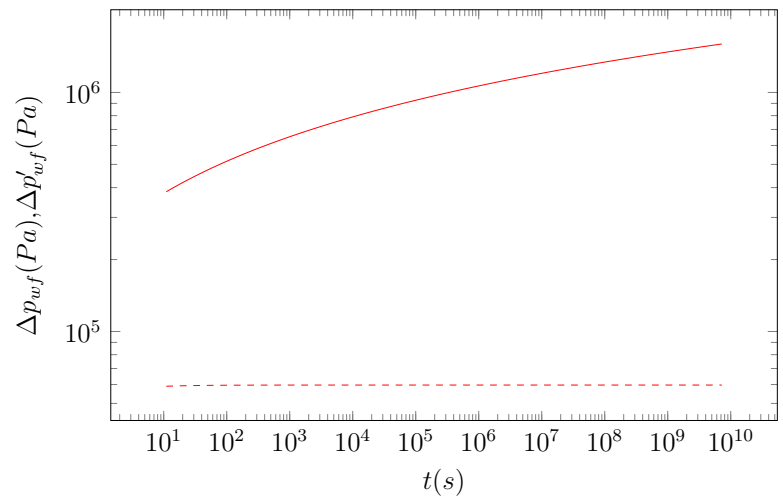

Figura 3: Gráfico diagnóstico para o Caso 1.

uma maior resistência ao escoamento. Tal comportamento pode ser observado na Figura 4 , notando que a inclinação da reta associada ao transiente muda conforme a permeabilidade é alterada.

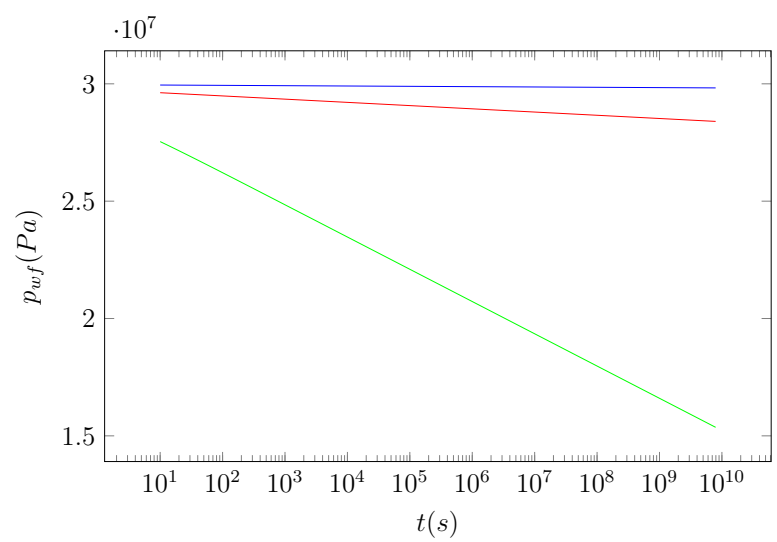

Figura 4: Gráfico especializado, Caso 1: $k=10^{-14} \mathrm{~m}^{2} \mathrm{em}$ verde, $k=10^{-13} \mathrm{~m}^{2}$ em vermelho e $k=10^{-12} \mathrm{~m}^{2}$ em azul.

Em se tratando do gráfico diagnóstico, na Figura 5 é possível notarmos que todas as derivadas de Bourdet são representadas aproximadamente por linhas retas horizontais, referentes ao escoamento transiente. Contudo, esses patamares encontram-se em diferentes posições verticais devido à variação da permeabilidade.

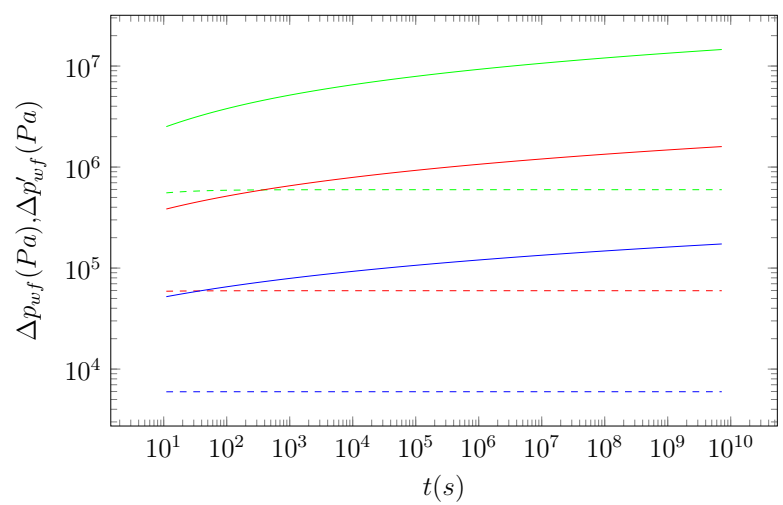

Figura 5: Gráfico diagnóstico, Caso 1: $k=10^{-14} \mathrm{~m}^{2} \mathrm{em}$ verde, $k=10^{-13} \mathrm{~m}^{2}$ em vermelho e $k=10^{-12} \mathrm{~m}^{2}$ em azul. 
As Figuras 6 e 7 trazem resultados em gráficos especializado e diagnóstico para diferentes valores da viscosidade do fluido. O efeito da variação da viscosidade é o inverso do relativo à permeabilidade, de modo que quanto maiores forem os valores da viscosidade, maiores serão as quedas de pressão no poço produtor para que ocorra o escoamento para uma vazão constante (Figura 6). Também percebemos que as derivadas seguem sendo aproximadas por linhas retas horizontais, características do sistema poço-reservatório considerado (Figura 7).

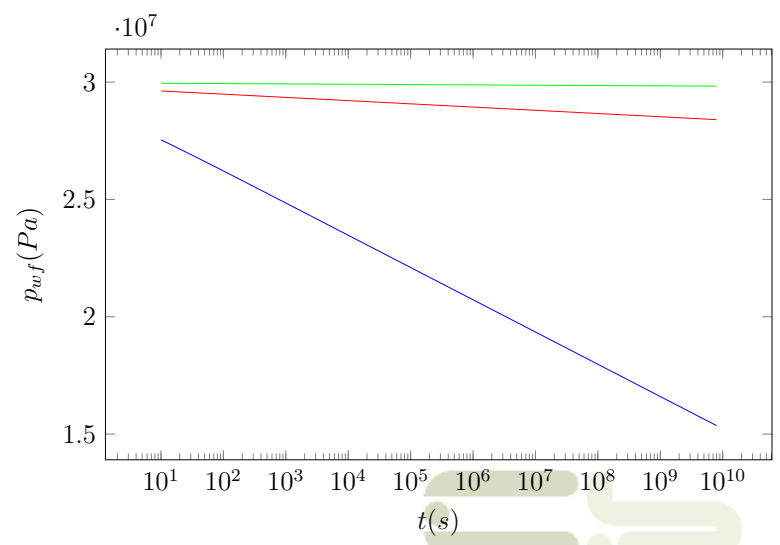

Figura 6: Gráfico especializado, Caso 1: $\mu=10^{-4} \mathrm{~Pa} \cdot \mathrm{s}$ em verde, $\mu=10^{-3} \mathrm{~Pa} \cdot \mathrm{s}$ em vermelho e $\mu=10^{-2} \mathrm{~Pa} \cdot \mathrm{s}$ em azul.

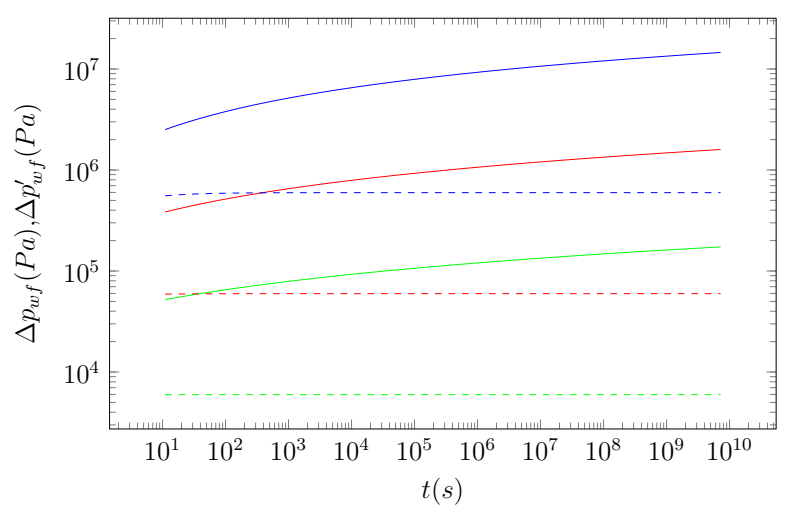

Figura 7: Gráfico diagnóstico, Caso 1: $\mu=10^{-4} \mathrm{~Pa} \cdot \mathrm{s}$ em verde, $\mu=10^{-3} \mathrm{~Pa} \cdot \mathrm{s}$ em vermelho e $\mu=10^{-2} \mathrm{~Pa} \cdot \mathrm{s}$ em azul.

As Figuras 8 e 9 contêm, respectivamente, os gráficos especializado e diagnóstico para a situação na qual variamos os valores da porosidade. Para os maiores valores da porosidade existirá mais fluido disponível para a produção e a queda de pressão no poço se tornará menor para uma vazão fixa. Notamos que a alteração na porosidade não influencia na inclinação da reta relativa ao regime transiente, presente na Figura 8, e que há uma sobreposição das curvas representando as derivadas na Figura 9

\subsection{Resultados para o Caso 2}

Os dados utilizados para encontrar soluções para o sistema poço-reservatório do Caso 2 podem ser vistos na

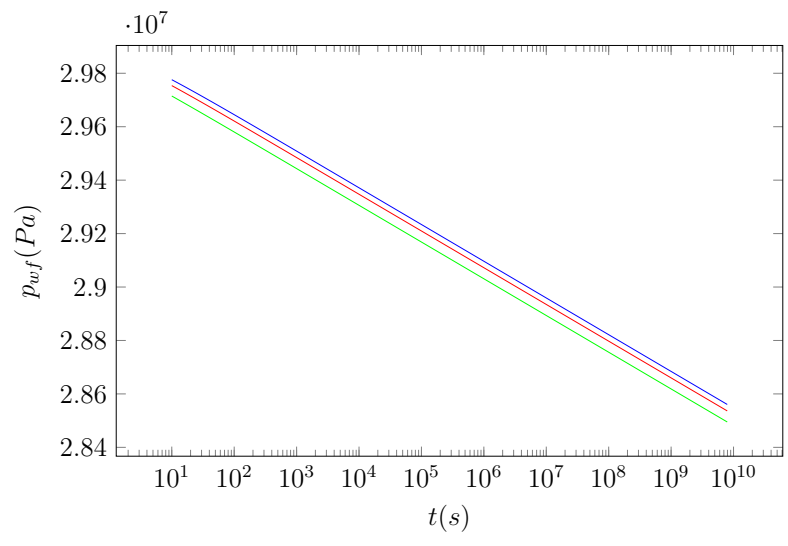

Figura 8: Gráfico especializado, Caso 1: $\phi=0,1$ em verde, $\phi=0,2$ em vermelho e $\phi=0,3$ em azul.

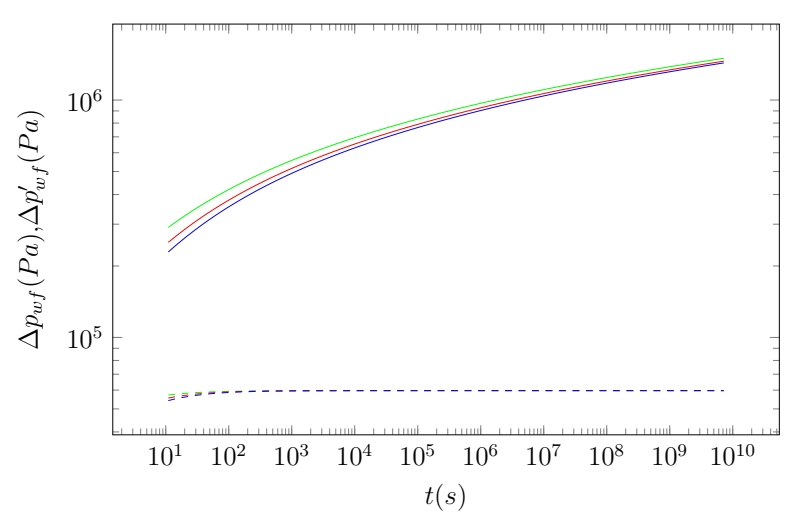

Figura 9: Gráfico diagnóstico, Caso 1: $\phi=0,1$ em verde, $\phi=$ 0,2 em vermelho e $\phi=0,3 \mathrm{em}$ azul.

Tabela 3. No presente caso são incorporados os efeitos de fronteira. Podemos notar que houve uma redução na vazão em relação ao Caso 1, pois agora o reservatório é finito e possui fronteiras definidas, limitando a quantidade fluido a ser produzida, diferentemente do Caso 1, cuja capacidade de produção depende da difusividade hidráulica, $\eta$.

Tabela 3 Parâmetros do Caso 2

\begin{tabular}{|c|c|}
\hline Dado & Valor \\
\hline$B$ & $1,50 \mathrm{~m}^{3} / \mathrm{std} \mathrm{m}^{3}$ \\
\hline$c$ & $1,00^{-8} \mathrm{~Pa}^{-1}$ \\
\hline$c_{\phi}$ & $1,00^{-8} \mathrm{~Pa}^{-1}$ \\
\hline$h$ & $20,00 \mathrm{~m}$ \\
\hline$k$ & $10,00^{-13} \mathrm{~m}^{2}$ \\
\hline$N$ & 10 \\
\hline$p_{i}$ & $30,00 \times 10^{6} \mathrm{~Pa}$ \\
\hline$Q_{s c}$ & $10,00^{-9} \mathrm{std} \mathrm{\textrm {m } ^ { 3 } / \mathrm { s }}$ \\
\hline$r$ & $0,10 \mathrm{~m}$ \\
\hline$r_{e}$ & $100,00 \mathrm{~m}$ \\
\hline$r_{w}$ & $0,10 \mathrm{~m}$ \\
\hline$\mu$ & $0,01 \mathrm{~Pa} \cdot \mathrm{s}$ \\
\hline$\phi$ & 0,20 \\
\hline
\end{tabular}

A Figura 10 apresenta o gráfico diagnóstico com a variacão da queda de pressão para o reservatório con- 
siderado no Caso 2. É importante observar que por se tratar de um reservatório fechado, para uma dada região os resultados para a queda de pressão irão diferir dos do Caso 1, devido ao início dos efeitos de fronteira. Isso fica mais evidente se olharmos a derivada de Bourdet, que não mais apresenta somente um patamar e passa a ter um crescimento representado por uma reta vertical inclinada. Após transcorrido um determinado período tempo, o reservatório deixa de se comportar como se fosse infinito e o escoamento passa por um regime de transição, conhecido como regime transiente tardio, para, em seguida, entrar no regime denominado pseudopermanente [1].

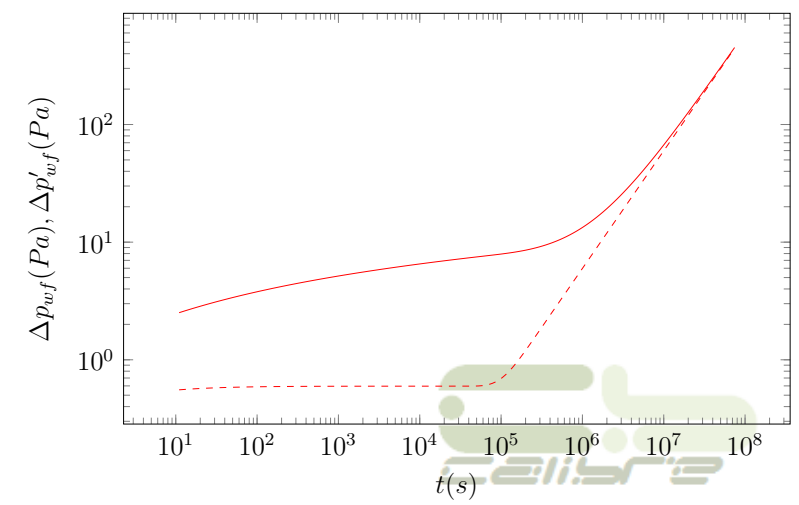

Figura 10: Gráfico diagnóstico para o Caso 2.

O efeito da variação do raio externo, para o Caso 2, pode ser percebido nos gráficos especializado e diagnóstico das Figuras 11 e 12. No gráfico especializado vemos que as soluções estão sobrepostas até que o efeito de fronteira ocorra, iniciando com o reservatório cilíndrico correspondente ao menor raio externo. A partir do gráfico diagnóstico, constatamos que as derivadas estão sobrepostas no regime transiente e se separam a partir do momento em que o efeito de fronteira começa a influenciar o escoamento. Também verificamos a sobreposição das curvas para $\Delta p_{w f}(P a)$ e $\Delta p_{w f}^{\prime}(P a)$ até que os efeitos de fronteira comecem a se pronunciar, acarretando na separação de cada uma das curvas.

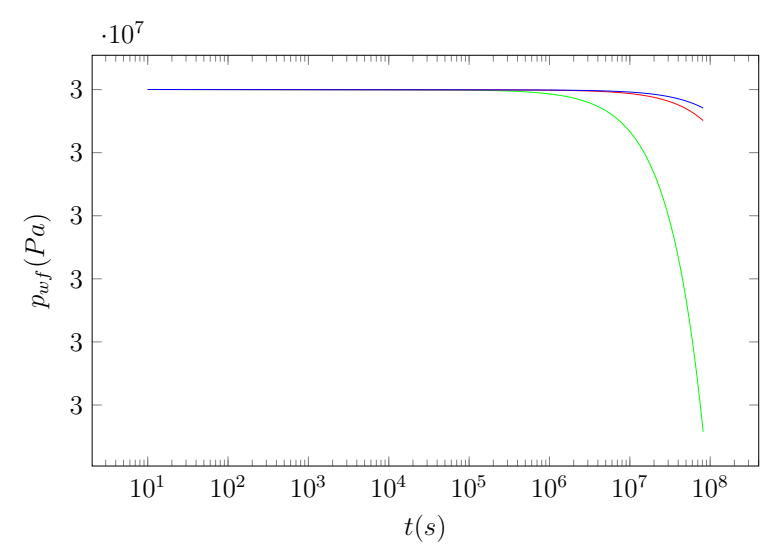

Figura 11: Gráfico especializado, Caso 2: $r_{e}=30 \mathrm{~m}$ em verde, $r_{e}=100 \mathrm{~m}$ em vermelho e $r_{e}=130 \mathrm{~m}$ em azul.

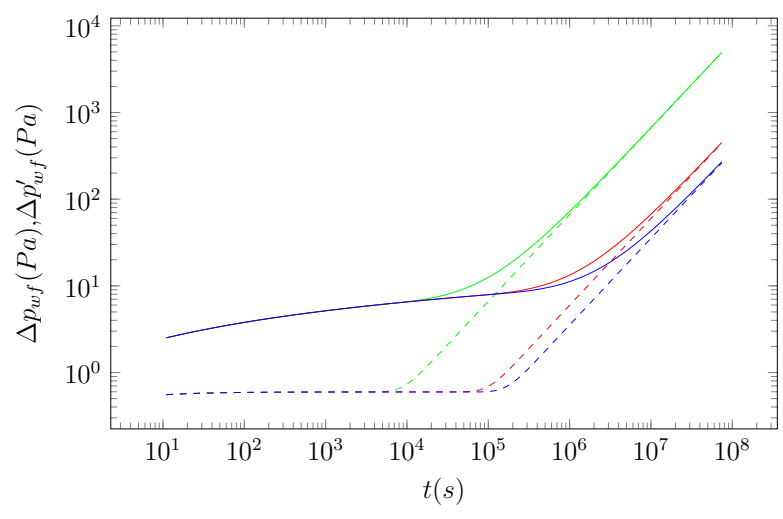

Figura 12: Gráfico diagnóstico, Caso 2: $r_{e}=30 \mathrm{~m}$ em verde, $r_{e}=100 \mathrm{~m}$ em vermelho e $r_{e}=130 \mathrm{~m}$ em azul.

Assim como o raio externo, a porosidade também modifica o tempo de transicão entre os regimes de escoamento transiente para o pseudopermanente. Através da análise das Figuras 13 e 14, vemos que os menores valores da porosidade antecipam a passagem para o regime pseudopermanente. Tal efeito é uma consequência da alteração do valor $\eta$ quando variamos a porosidade $\phi$.

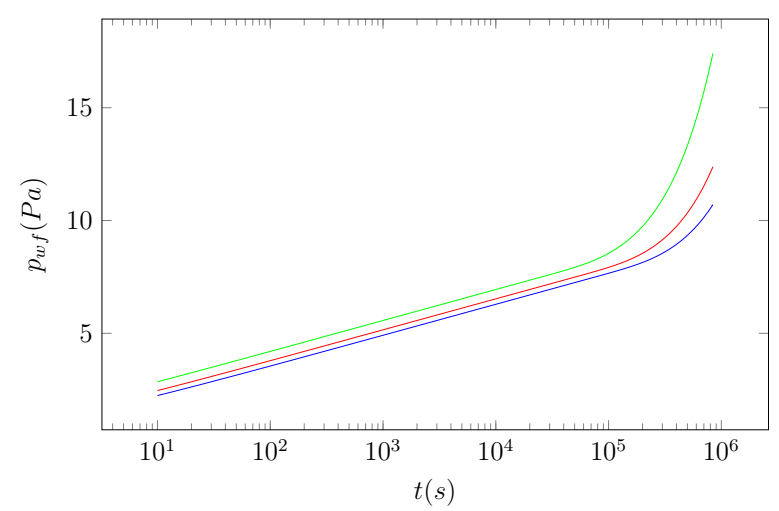

Figura 13: Variação de pressão, Caso 1: $\phi=0,1$ em verde, $\phi=0,2$ em vermelho e $\phi=0,3$ em azul.

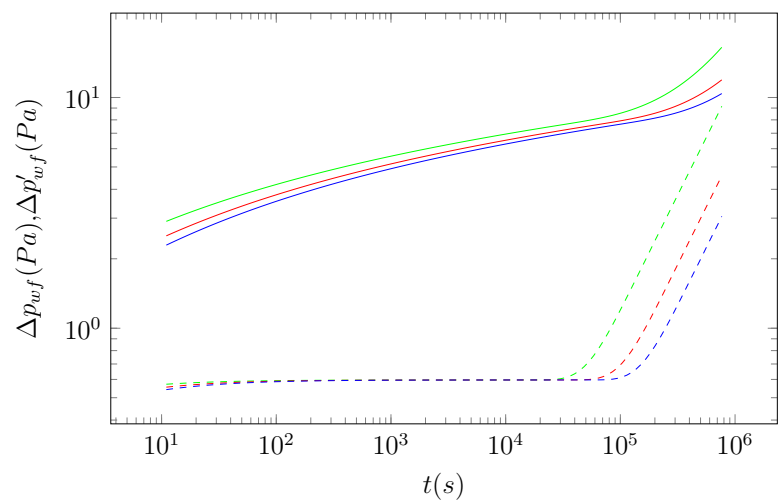

Figura 14: Gráfico diagnóstico, Caso 2: $\phi=0,1$ em verde, $\phi=0,2$ em vermelho e $\phi=0,3 \mathrm{em}$ azul. 


\subsection{Resultados para o Caso 3}

Nossa atenção agora está voltada para os efeitos de estocagem de poço e de dano à formação, que ocorrem nos primeiros instantes da produção. Os parâmetros utilizados para a previsão teórica da variação da perda de pressão no reservatório, para o Caso 3, são mostrados na Tabela 4

Tabela 4 Parâmetros do Caso 3

\begin{tabular}{cl}
\hline Dado & Valor \\
\hline$B$ & $1,50 \mathrm{~m}^{3} / \mathrm{std} \mathrm{m}^{3}$ \\
$c$ & $1,00^{-8} \mathrm{~Pa}^{-1}$ \\
$c_{\phi}$ & $1,00^{-8} \mathrm{~Pa}^{-1}$ \\
$C$ & $10,00^{-3} \mathrm{~m}^{3} / \mathrm{Pa}$ \\
$h$ & $20,00 \mathrm{~m}$ \\
$k$ & $10,00^{-13} \mathrm{~m}^{2}$ \\
$N$ & 10 \\
$p_{i}$ & $30,00 \times 10^{6} \mathrm{~Pa}$ \\
$Q_{s c}$ & $10,00^{-3} \mathrm{std}^{3} / \mathrm{s}$ \\
$r$ & $0,10 \mathrm{~m}$ \\
$r_{w}$ & $0,10 \mathrm{~m}$ \\
$S$ & 5,00 \\
$\mu$ & $0,01 \mathrm{~Pa} \cdot \mathrm{s}$ \\
$\phi$ & 0,20
\end{tabular}

A curva característica do comportamento da queda de pressão, para um reservatório com as características do Caso 3, é apresentada no gráfico diagnóstico da Figura 15. Chamamos a atenção para o comportamento diferenciado da derivada de Bourdet, que evidencia claramente os efeitos do dano à formação e da estocagem no poço. Os seus valores atingem um máximo e, em seguida, decrescem até atingir um valor que permanece praticamente constante.

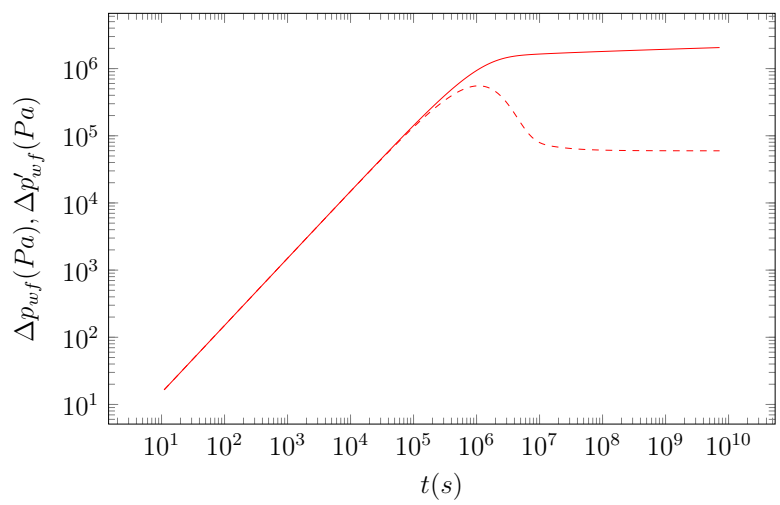

Figura 15: Gráfico diagnóstico para o Caso 3.

O efeito do dano à formação modifica a região da interface poço-reservatório, criando um efeito de película que acaba alterando as características do escoamento, pois resulta em uma maior resistência ao escoamento do fluido [1]. As Figuras 16 e 17 apresentam resultados para a variação da intensidade do dano à formação. Um aumento do coeficiente de dano gera uma maior restrição ao escoamento, sendo necessário um $\Delta p_{w f}$ maior para que a vazão de produção pré-fixada seja preservada. Os resultados dos gráficos corroboram tal previsão. A derivada de Bourdet também é influenciada pelo fenômeno de estocagem, que depende do valor de $k$, de forma que quanto menor a permeabilidade maior será a duração do efeito de estocagem no poço. A permeabilidade da região danificada também será menor do aquela fora da região próxima da interface poço-reservatório.

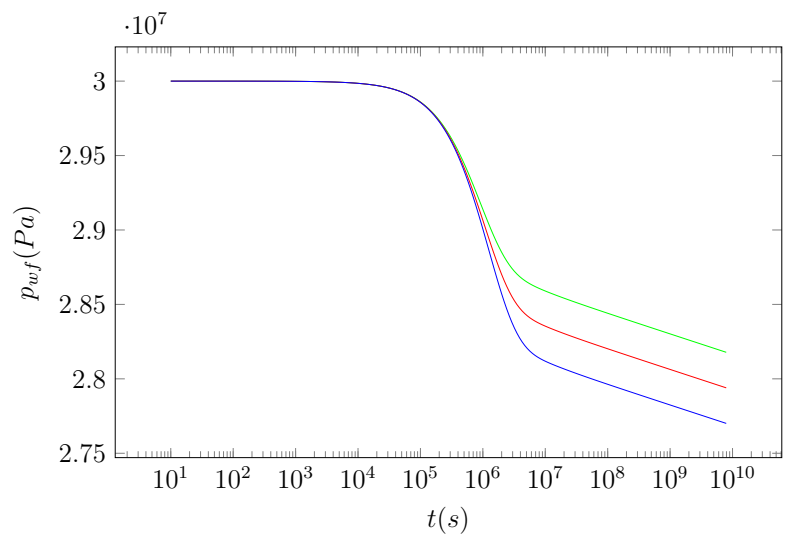

Figura 16: Gráfico especializado, Caso 3: $S=3$ em verde, $S=5$ em vermelho e $S=7$ em azul.

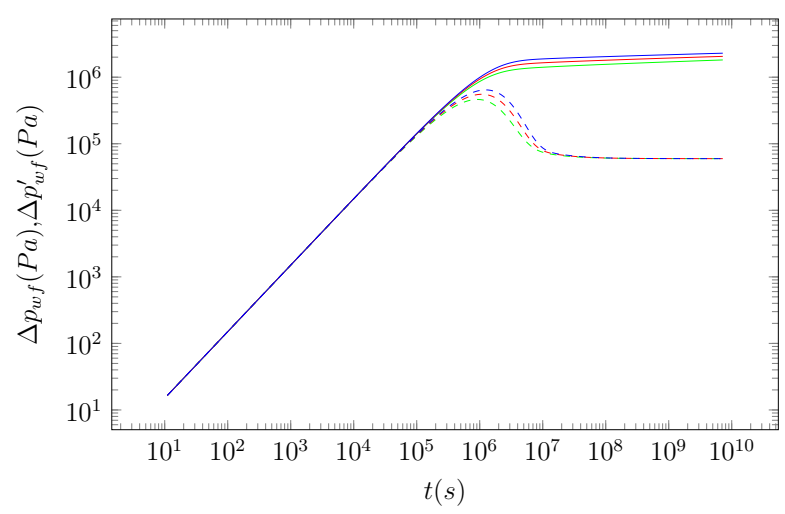

Figura 17: Gráfico diagnóstico, Caso 3: $S=3$ em verde, $S=5$ em vermelho e $S=7$ em azul.

Quando do início da produção, em um sistema sob o efeito da estocagem, a variação de pressão está diretamente relacionada com as características do escoamento do fluido estocado no poço, sendo muito pequena a contribuição devida ao escoamento no interior do reservatório. Com o passar do tempo e a saída do fluido estocado, a vazão decorrente do movimento do fluido contido no interior do reservatório começará a subir. A partir de um determinado instante de tempo, a estocagem não interferirá mais na produtividade e a vazão passará a ser dominada pelo escoamento no reservatório [1]. Da Figura 18 podemos constatar, de maneira bem clara, a tardia queda de pressão causada pelo aumento do coeficiente de estocagem. 


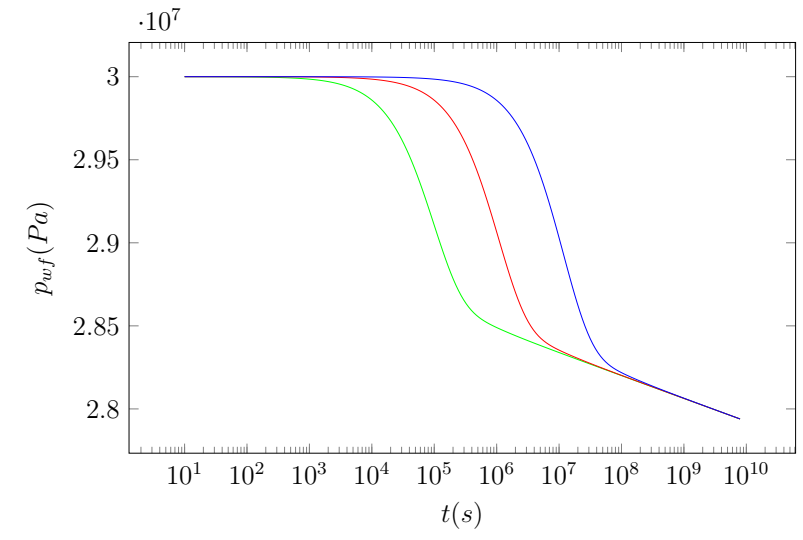

Figura 18: Gráfico especializado, Caso 3: $C=10^{-4} \mathrm{~m}^{3} / \mathrm{Pa}$ em verde, $C=10^{-3} \mathrm{~m}^{3} / \mathrm{Pa}$ em vermelho e $C=10^{-2} \mathrm{~m}^{3} / \mathrm{Pa}$ em azul.

Ainda na Figura 18, a linha azul, correspondente ao maior coeficiente de estocagem $\left(C=0,01 \mathrm{~m}^{3} / \mathrm{Pa}\right)$ começará a apresentar uma queda de pressão significativa após aproximadamente $10^{5} \mathrm{~s}$, embora as curvas determinadas com valores menores desse coeficiente já tenham tido uma queda de pressão acentuada para instantes de tempo anteriores. Como pode ser visto, as curvas em vermelho $\left(C=0,001 \mathrm{~m}^{3} / \mathrm{Pa}\right)$ e em verde $(C=$ $\left.0,001 \mathrm{~m}^{3} / \mathrm{Pa}\right)$ mostram que a queda de pressão tem início decorridos aproximadamente $10^{4} \mathrm{~s}$ e $10^{3} \mathrm{~s}$, respectivamente. É interessante notar que após cerca de $10^{8} \mathrm{~s}$ as três curvas já se encontram sobrepostas umas as outras. Nesse momento, a vazão oriunda da estocagem já não estará mais contribuindo para a produção no reservatório. No caso do gráfico diagnóstico, Figura 19, vemos que as curvas encontram-se deslocadas. Portanto, percebemos que à medida que aumentamos o coeficiente de estocagem haverá um atraso na contribuição do reservatório na produção, evidenciada pelo demora no começo do regime transiente em meio poroso.

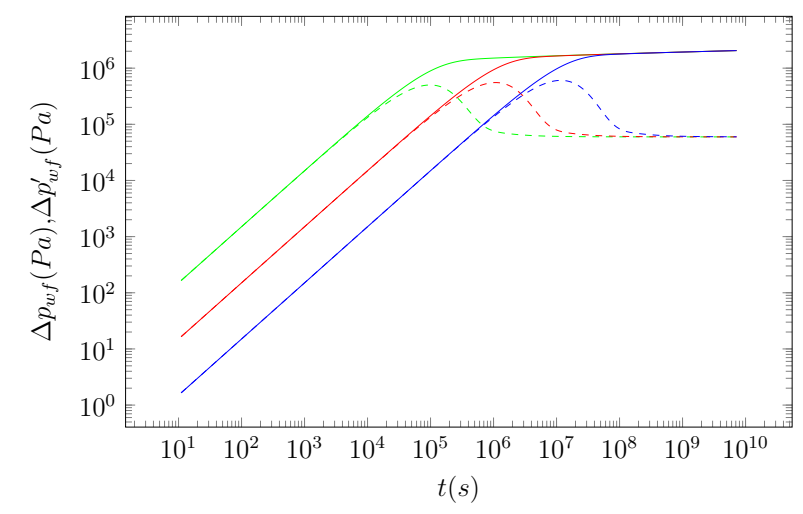

Figura 19: Gráfico diagnóstico, Caso 3: $C=10^{-4} \mathrm{~m}^{3} / \mathrm{Pa}$ em verde, $C=10^{-3} \mathrm{~m}^{3} / \mathrm{Pa}$ em vermelho e $C=10^{-2} \mathrm{~m}^{3} / \mathrm{Pa}$ em azul.

Nas Figuras 20 e 21, respectivamente para os gráficos especializado e diagnóstico, podemos quantificar a importância da variação do número de termos $N$ no algoritmo de Stehfest. Apesar das curvas estarem pratica- mente sobrepostas, na literatura encontramos relatos de casos nos quais diferentes valores de $N$ interferiram fortemente na acurácia dos resultados para outros sistemas poço-reservatório 22 .

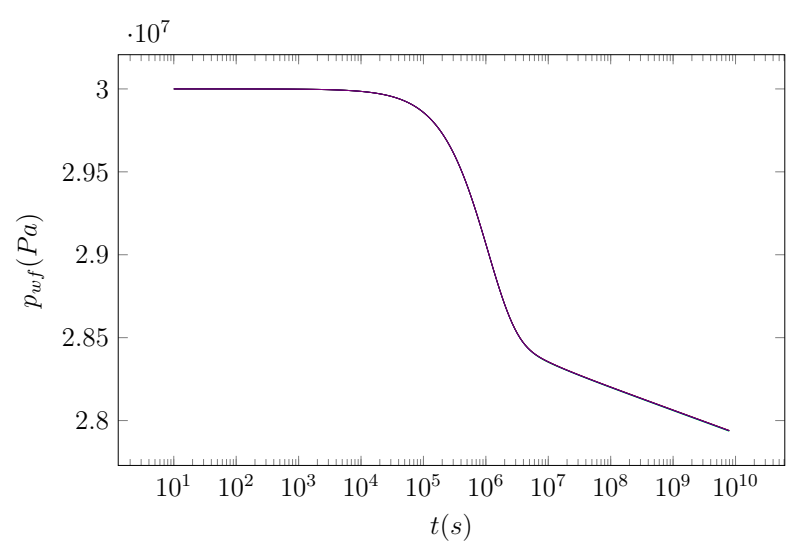

Figura 20: Gráfico especializado, Caso 3: $N=8$ em verde, $N=10$ em vermelho, $N=12 \mathrm{em}$ azul, $N=14$ em preto e $N=16$ em roxo.

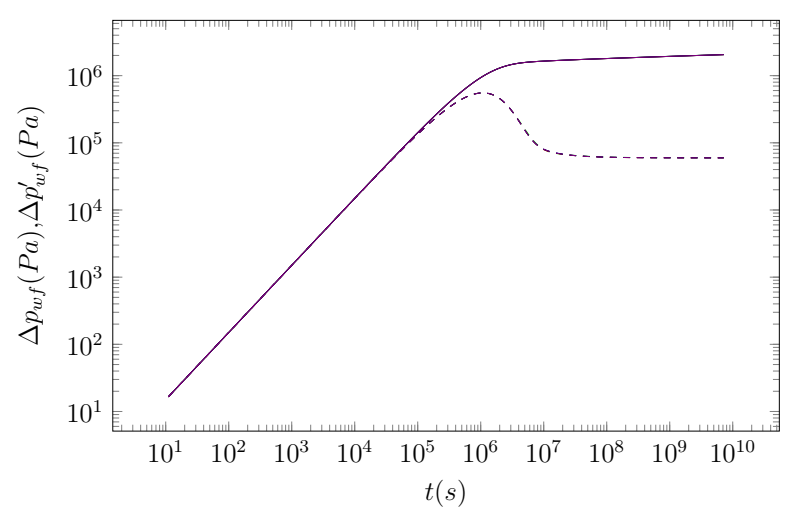

Figura 21: Gráfico diagnóstico, Caso 3: $N=8$ em verde, $N=$ 10 em vermelho, $N=12 \mathrm{em}$ azul, $N=14$ em preto e $N=16$ em roxo.

\subsection{Resultados para o Caso 4}

Na Tabela 5 encontram-se os dados utilizados nas simulações levando em conta o Caso 4. Nele, a correção sugerida por Chen e Raghavan [7] foi utilizada no algoritmo de Stehfest, visando a se reproduzir corretamente o registro da variação de pressão em uma sequência de produção e fechamento do poço.

A variação da queda de pressão pode, então, ser vista na Figura 22. Este sistema poço-reservatório possui as mesmas características de escoamento que as do Caso 3. No entanto, nos testes realizados, na fase de produção, o poço é mantido aberto por um determinado tempo, $t_{p}$, e a vazão de produção é mantida constante. Esse procedimento (a produção) causará uma diminuição da pressão no poço e no reservatório. Após transcorrido o tempo $t_{p}$,

CALIBRE Vol.5, Suplemento - Dezembro, 2020. 
Tabela 5 Parâmetros do Caso 4

\begin{tabular}{|c|c|}
\hline Dado & Valor \\
\hline$B$ & $1,50 \mathrm{~m}^{3} / \mathrm{std} \mathrm{\textrm {m } ^ { 3 }}$ \\
\hline$c$ & $1,00^{-8} \mathrm{~Pa}^{-1}$ \\
\hline$c_{\phi}$ & $1,00^{-8} \mathrm{~Pa}^{-1}$ \\
\hline$C$ & $10^{-3} \mathrm{~m}^{3} / \mathrm{Pa}$ \\
\hline$h$ & $20,00 \mathrm{~m}$ \\
\hline$k$ & $10^{-13} \mathrm{~m}^{2}$ \\
\hline$N$ & 10 \\
\hline$p_{i}$ & $30,00 \times 10^{6} \mathrm{~Pa}$ \\
\hline$Q_{s c}$ & $10^{-3} \mathrm{std} \mathrm{m}^{3} / \mathrm{s}$ \\
\hline$r$ & $0,10 \mathrm{~m}$ \\
\hline$r_{w}$ & $0,10 \mathrm{~m}$ \\
\hline$S$ & 5,00 \\
\hline$t_{p}$ & $4320000 \mathrm{~s}$ \\
\hline$\mu$ & $0,01 \mathrm{~Pa} \cdot \mathrm{s}$ \\
\hline$\phi$ & 0,20 \\
\hline
\end{tabular}

o poço é fechado e a pressão tenderá a retornar à pressão inicial, antes da abertura do mesmo. Entretanto, isso não ocorrerá devido à perda de fluido durante a fase de estática (poço fechado).

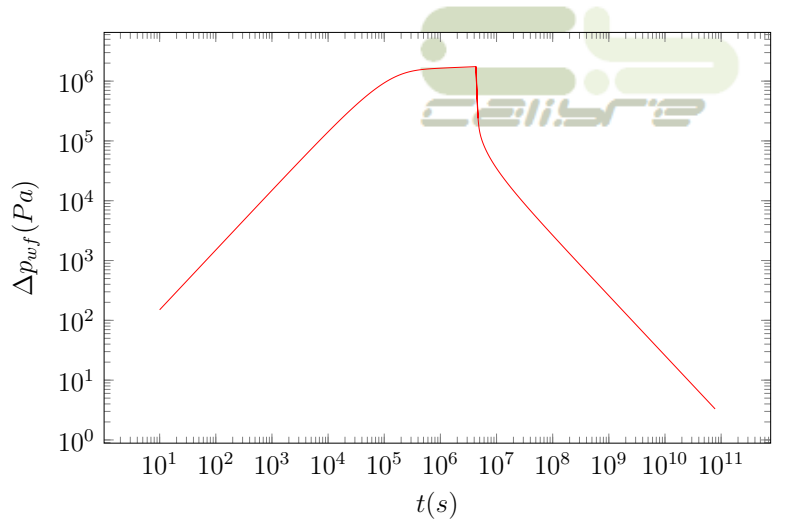

Figura 22: Variação de pressão, Caso 4.

Em contrapartida, a Figura 23 mostra como a variação de pressão no poço muda quando modificamos o de tempo de produção $t_{p}$. Notamos que as cuvas descrevem qualitativamente o mesmo tipo de comportamento, à exceção do tempo no qual se inicia o processo de recuperação de pressão.

Por outro lado, conforme já vimos, a modificação do coeficiente de estocagem $C$ altera o início da produção e, também, o comportamento da pressão no período de poço fechado, como pode ser visto na Figura 24. Portanto, quanto maior for o coeficiente $C$ maior será o tempo necessário para que aconteça o reequilíbrio de pressão.

No que diz respeito à variação dos valores da permeabilidade, o efeito de estocagem se torna mais significativo tanto na abertura quanto no fechamento do poço. No caso do fechamento do poço, o fluido oriundo do reservatório que já estava se deslocando, antes da etapa de fechamento, continuará a alcançar o poço mesmo após a interrupção da produção. Tal fato se reflete no gráfico da variação da pressão no poço, Figura 25.

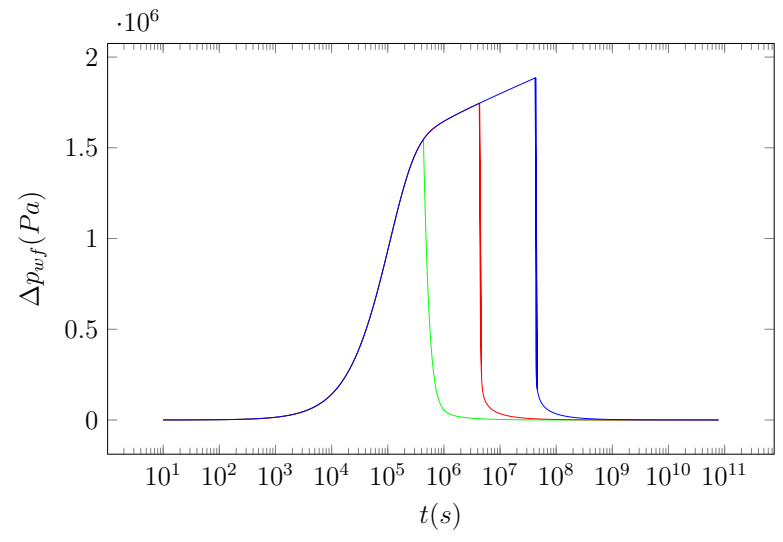

Figura 23: Variação de pressão, Caso 4: $t_{p}=43,210^{4} \mathrm{~s}$ em verde, $t_{p}=43,210^{5} \mathrm{~s}$ em vermelho e $t_{p}=43,210^{6} \mathrm{~s}$ em azul.

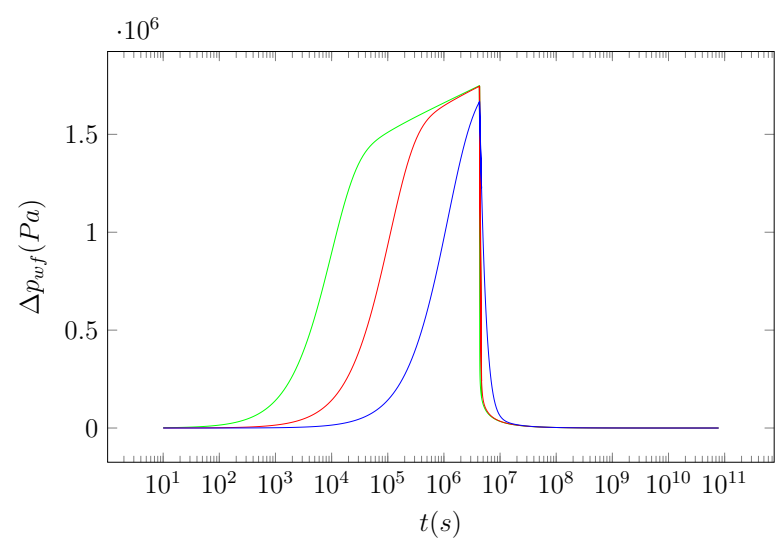

Figura 24: Variação de pressão, Caso 4: $C=10^{-4} \mathrm{~m}^{3} / \mathrm{Pa}$ em verde, $C=10^{-3} \mathrm{~m}^{3} / \mathrm{Pa}$ em vermelho e $C=10^{-2} \mathrm{~m}^{3} / \mathrm{Pa}$ em azul.

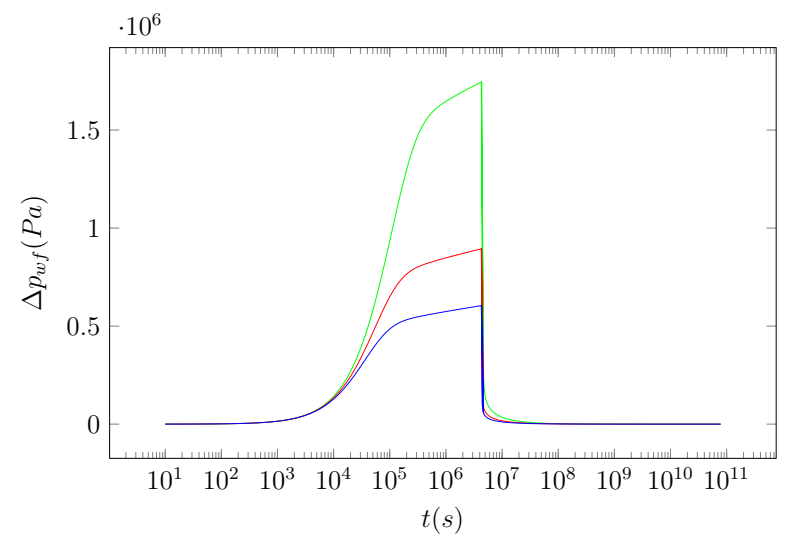

Figura 25: Variação de pressão, Caso $4: k=1 \times 10^{-13} \mathrm{~m}^{2} \mathrm{em}$ verde, $k=2 \times 10^{-13} \mathrm{~m}^{2}$ em vermelho e $k=3 \times 10^{-13} \mathrm{~m}^{2} \mathrm{em}$ azul.

\subsection{Resultados para o Caso 5}

No Caso 5, utilizamos como valores padrão $N=10$, $\omega=0,01$ e $\lambda=510^{-8}$. A Figura 26 mostra o gráfico diagnóstico com as curvas representativas do presente caso. Para um reservatório naturalmente fraturado, dois fatores, $\omega$ e $\lambda$, são utilizados na caracterização da mudança 
de comportamento do escoamento no reservatório, causada pela presença das fraturas [28. São consideradas duas porosidades distintas, uma para a fratura e outra para a matriz porosa do reservatório. Em se tratando das curvas da Figura 26, verificamos claramente a região de transição, na qual ambas a fratura e a matriz porosa influenciam o escoamento. No que diz respeito à variação de pressão, percebemos uma mudança através do surgimento de uma região de transição entre os dois trechos que se assemelham às curvas dos casos dos reservatórios homogêneos. Já para a curva da derivada de Bourdet, notamos a formação de um "vale" separando as duas regiões mencionadas previamente.

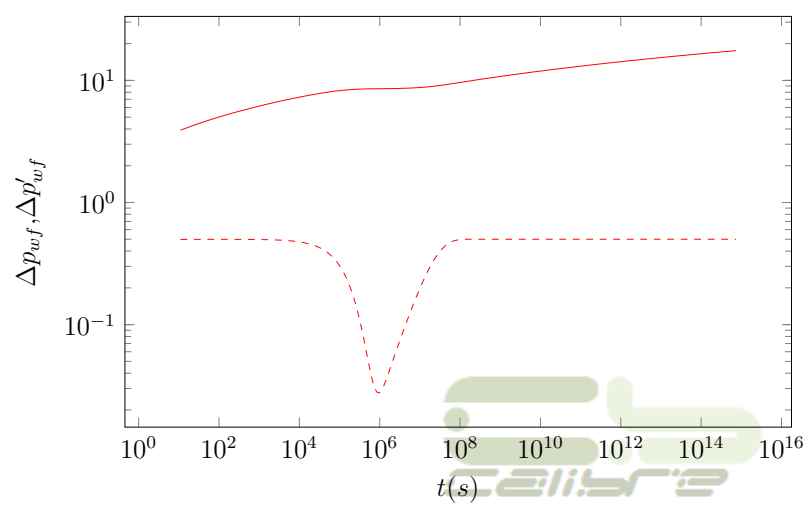

Figura 26: Gráfico diagnóstico, Caso 5.

Na Figura 27, temos o gráfico especializado com os resultados para a queda de pressão em função da variação dos valores de $\lambda$. Das curvas da figura fica claro que ele influencia na duração da transição, entre as regiões de predomínio do escoamento na fratura e na matriz porosa. Portanto, quanto maior o seu valor mais cedo ocorrerá a transição. Segundo Warren e Root 28, quando $\lambda \rightarrow \infty$ o reservatório tenderá a se comportar como um meio poroso homogêneo.

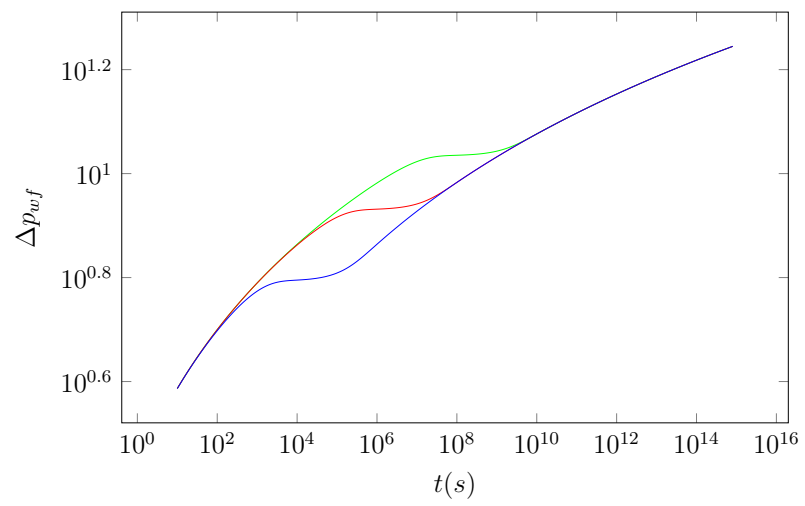

Figura 27: Variação de pressão, Caso 5: $\lambda=5 \times 10^{-10} \mathrm{em}$ verde, $\lambda=5 \times 10^{-8}$ em vermelho e $\lambda=5 \times 10^{-6}$ em azul.

Prosseguindo, no gráfico diagnóstico da Figura 28 verificamos que ao variarmos o valor de $\lambda$ a posição do "vale" sofre uma translação. Um comportamento similar pode ser obtido a partir da coleta de dados de campo e, por meio da resolução de um problema inverso, nos auxiliar na caracterização do sistema poço-reservatório através da determinação de uma estimativa para o valor de $\lambda$.

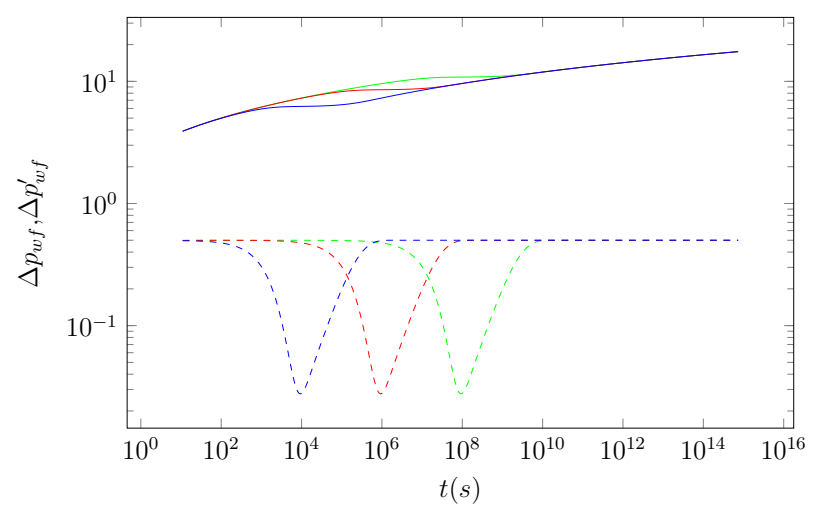

Figura 28: Gráfico diagnóstico, Caso 5: $\lambda=5 \times 10^{-10} \mathrm{em}$ verde, $\lambda=5 \times 10^{-8}$ em vermelho e $\lambda=5 \times 10^{-6}$ em azul.

Finalmente, nas Figuras 29 e 30 vemos os resultados determinados para diferentes valores de $\omega$. No nosso entender, é evidente o seu papel na transição entre os regimes, sendo que a extensão das zonas de transição será maior para os menores valores de $\omega$ (Figura 29p. Conforme discutido em Warren e Root 28, quando $\omega \rightarrow 1$ a porosidade primária (da fratura) terá uma menor influência, evidenciando o efeito da porosidade secundária (da matriz) e, então, o reservatório apresentará o comportamento de um meio homogêneo. Portanto, quanto menor o parâmetro $\omega$, maior será a influência da fratura. Das curvas das derivadas de Bourdet (Figura 30, notamos que a curva associada ao valor $\omega=0,1$ possui um comportamento mais próximo do verificado no Caso 1, onde o reservatório é infinito e não contém nenhuma fratura.

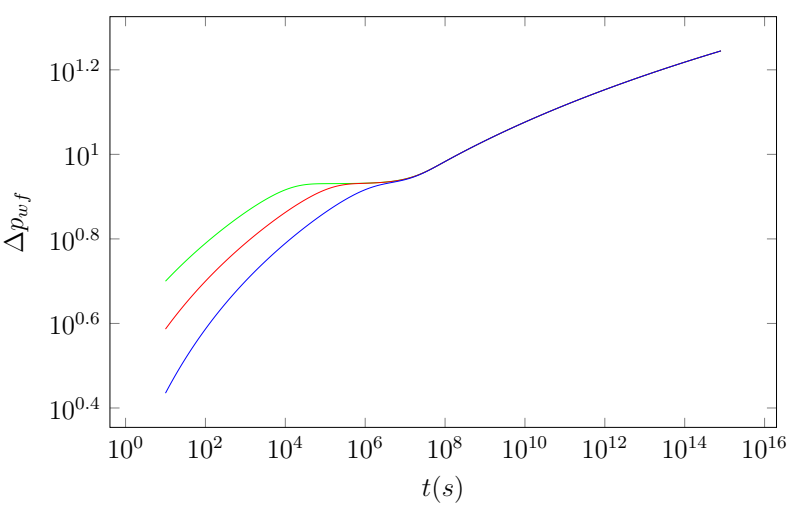

Figura 29: Variação de pressão, Caso 5: $\omega=10^{-3}$ em verde, $\omega=10^{-2}$ em vermelho e $\omega=10^{-1} \mathrm{em}$ azul.

\section{Conclusões}

O principal objetivo deste trabalho foi atingido, com a criação de uma biblioteca de códigos numéricos, no con- 


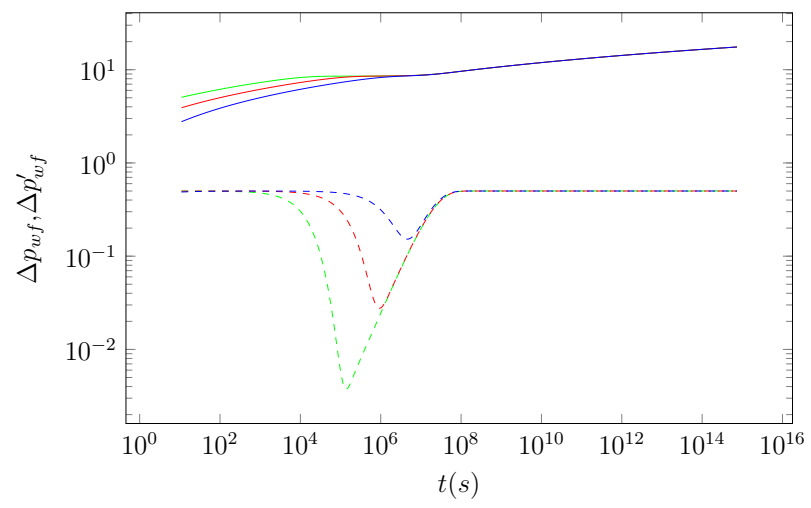

Figura 30: Gráfico diagnóstico, Caso 5: $\omega=10^{-3}$ em verde, $\omega=10^{-2}$ em vermelho e $\omega=10^{-1}$ em azul.

texto da Análise de Testes de Pressão, baseados em algumas das mais conhecidas soluções analíticas disponíveis na literatura para a determinação da variação de pressão no poço produtor. Esses programas computacionais serão utilizados na verificação dos simuladores desenvolvidos no Laboratório de Modelagem Multiescala e Transporte de Partículas da Universidade do Estado do Rio de Janeiro. Por fim, os mesmos também podem ser empregados na caracterização dos sistemas poço-reservatório que se enquadrem nos casos aqui analisados, após uma ampliação dos códigos.

No que diz respeito à implementação dos códigos numéricos, podemos destacar a eliminação de pequenas oscilações nas derivadas de Bourdet e a ampliação da faixa de valores da frequência $s$, relevante na determinação da pressão no poço para tempos curtos, essenciais no âmbito da análise dos testes de pressão. A melhoria na captura dos resultados ocorreu pela utilização de valores de dupla precisão nas rotinas pesquisadas na literatura para o cálculo das funções modificadas de Bessel.

Em relação aos resultados obtidos, eles reproduziram satisfatoriamente os resultados esperados e conhecidos na literatura. Portanto, entendemos que logramos êxito na captura dos efeitos de fronteira, da estocagem no poço, do dano à formação, dos testes de produção e estática e da presença de fraturas naturais no conjunto poço-reservatório.

\section{$7 \quad$ Agradecimentos}

Os autores gostariam de agradecer o suporte financeiro fornecido pela FAPERJ.

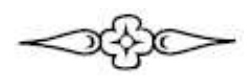

CALIBRE - Revista Brasiliense de Eng enharia e Fisica Aplicada, ISSN 2526-4192. Livre dire ito de cópia de acordo com os princípios estabelecidos pela Creative Common.
Referências

[1] Bourdet, D. (2002). Well Test Analysis: the Use of Advanced Interpretation Models. Handbook of Petroleum Exploration and Production 3. Elsevier, Amsterdam.

[2] Adameka, V.; Vales, F.; Cerv, J. (2016). Numerical Laplace inversion in problems of elastodynamics: Comparison of four algorithms. Advances in Engineering Software, $113,1-10$.

[3] Awais, M. (2015). Applications of the numerical inversion of the Laplace transform to unsteady problems of the third grade fluid. Applied Mathematics and Computation, 250, 228-234.

[4] Bourdet, D.; Ayuob, J. A.; Pirard, Y. M. (1989). Use of pressure derivative in well-test interpretation. SPE Formation Evaluation, 19, 293-302.

[5] Campos, R. G.; Huet, A. (2018). Numerical inversion of the Laplace transform and its application to fractional diffusion. Applied Mathematics and Computation, 327, 70-234.

[6] Chaudhry, A. U. (2004). Oil Well Testing Handbook. Gulf Professional Publishing, Burlington.

[7] Chen, C. C.; Raghavan, R. (1996). An approach to handle discontinuities by the Stehfest algorithm. SPE Journal, 363.

[8] Chen, S.; Liu, F.; Turner, I.; Hu, X. (2018). Numerical inversion of the fractional derivative index and surface thermal flux for an anomalous heat conduction model in a multi-layer medium. Applied Mathematical Modelling, 59, 514-526.

[9] Dake, L. P. (2001). The Practice of Reservoir Engineering (Revised Edition). Amsterdam, The Netherlands: Elsevier, Developments in Petroleum Science, 36.

[10] Dandekar, A. Y. (2013). Petroleum Reservoir Rock and Fluid Properties. USA: CRC Press.

[11] Deleruyelle, F.; Bui, T. A.; Wong, H.; Dufour, N.; Tran, D. K.; Zhang, X. S. (2016). Analytical study of the post-closure behaviour of a deep tunnel in a porous creeping rock mass. Comptes Rendus Mecanique, 344, 649-660.

[12] Du, X.; Lu, Z.; Li, D; Xu, Y.; Li, P.; Lu, D. (2019). A novel analytical well test model for fractured vuggy carbonate reservoirs considering the coupling between oil flow and wave propagation. Journal of Petroleum Science and Engineering, 173, 447-461.

[13] Ertekin, T.; Abou-Kassem, J.; King, G. (2001). Basic Applied Reservoir Simulation. Society of Petroleum Engineers, Richardson, USA. 
[14] Fanchi, J. R. (2010). Integrated Reservoir Asset Management. Gulf Professional Publishing, Boston.

[15] Hassanzadeh, H.; Pooladi-Darvish, M. (2007). Comparison of different numerical Laplace inversion methods for engineering applications. Applied Mathematics and Computation, 189, 1966-1981.

[16] Huang, Y.; Cheng, S.; Yu, H.; He, Y.; Lin, B.; Feng, N. (2017). A semianalytical approach to estimate fracture closure and formation damage of vertically fractured wells in tight gas reservoir. Journal of Petroleum Science and Engineering, 150, 85-90.

[17] Kamal, M. M. and Morsy, S. and Suleen, F. and Pan, Y. and Dastan, A. and Stuart, M. R. and Mire, E. and Zakariya, Z. (2019). Determination of in-situ reservoir absolute permeability under multiphaseflow conditions using transient well testing. SPE Reservoir Evaluation \& Engineering, 22 (1), 1-15.

[18] Kazemi, H. (1969). Pressure transient analysis of naturally fractured reservoirs with uniform fracture distribution. SPE Journal, 9 (4).

[19] Laribi, S.; Bertin, H.; Quintard, M. (1995). Twophase calculations and comparative flow experiments through heterogeneous orthogonal stratified systems. Journal of Petroleum Science and Engineering, 12 (3), 183-199.

[20] Li, Y.; Li, X.; Teng, S.; Wang, F.; Xu, D. (2014). A new changing wellbore storage model for pressure oscillation in pressure buildup test. Journal of Natural Gas Science and Engineering, 19, 350-357.

[21] New Mexico Tech (2018). Derivative Algorithms.

[22] Ozkan, E. (2008). Applied Mathematics of Fluid Flow in Porous Media.

[23] Pouladi, B. and Sharifi, M. and Akbari, M. R. and Hosseini-Nasab, S. M. (2019). A new numerical well testing approach: Application to characterization of complex fault structures. Petroleum, 5, (3) 295-302.

[24] Press, W. H. et al. (1992). Numerical Recipes in C, The Art of Scientific Computing, 2nd edition. Cambridge University Press, New York, USA.

[25] Qiu, K.; Li, H. (2018). A new analytical solution of the triple-porosity model for history matching and performance forecasting in unconventional oil reservoirs. SPE Journal, 23 (6), 1-20.

[26] Rosa, A. J.; Carvalho, R. S.; Xavier, J. A. (2006). Engenharia de Reservatórios de Petróleo. Interciência, Brasil.

[27] Stehfest, H. (1970). Algorithm 368: Numerical inversion of Laplace Transform. Communication of the ACM, 1, 47-49.
[28] Warren, J.; Root, P. (1963). The behavior of naturally fracture reservoirs. Society of Petroleum Engineers Journal, 3 (3), 245-255.

[29] Wendong, W.; Shahvali, M.; Yuliang, S. (2015). A semi-analytical fractal model for production from tight oil reservoirs with hydraulically fractured horizontal wells. Fuel, 158, 613-618.

[30] Zeng, F.; Zhao, G. (2008). Semianalytical model for reservoirs with Forchheimer's non-Darcy flow Society of Petroleum Engineers Reservoir Evaluation \& Engineering, 11 (2), 280-291. 